\title{
The star catalogue of Hevelius
}

\section{Machine-readable version and comparison with the modern Hipparcos Catalogue $^{\star}$}

\author{
F. Verbunt ${ }^{1}$ and R. H. van Gent ${ }^{2,3}$ \\ 1 Astronomical Institute, Utrecht University, PO Box 80 000, 3508 TA Utrecht, The Netherlands \\ e-mail: f.w.m.verbunt@uu.nl \\ 2 URU-Explokart, Faculty of Geosciences, Utrecht University, PO Box 80 115, 3508 TC Utrecht, The Netherlands \\ 3 Institute for the History and Foundations of Science, PO Box 80 000, 3508 TA Utrecht, The Netherlands
}

Received 6 January 2010 / Accepted 4 February 2010

\section{ABSTRACT}

\begin{abstract}
The catalogue by Johannes Hevelius with the positions and magnitudes of 1564 entries was published by his wife Elisabeth Koopman in 1690 . We provide a machine-readable version of the catalogue, and briefly discuss its accuracy on the basis of comparison with data from the modern Hipparcos Catalogue. We compare our results with an earlier analysis by Rybka (1984), finding good overall agreement. The magnitudes given by Hevelius correlate well with modern values. The accuracy of his position measurements is similar to that of Brahe, with $\sigma=2^{\prime}$ for longitudes and latitudes, but with more errors $>5^{\prime}$ than expected for a Gaussian distribution. The position accuracy decreases slowly with magnitude. The fraction of stars with position errors larger than a degree is $1.5 \%$, rather smaller than the fraction of 5\% in the star catalogue of Brahe.
\end{abstract}

Key words. astrometry - history and philosophy of astronomy

\section{Introduction}

Even though a major improvement on earlier work, the star catalogue produced by Tycho Brahe $(1598,1602)$, and re-edited by Kepler (1627), contains occasional large errors. Johannes Hevelius decided to produce a better and larger catalogue, which was printed after his death by his wife and collaborator Elisabeth Koopman (Hevelius 1690). The title page has 1687, the year in which the catalogue was printed, but publication followed only in 1690 . The extent of the contribution by Koopman to measuring the stars and producing the catalogue is not known; her presence on several images of instruments used by Hevelius suggests that it was significant. A brief but informative and wellillustrated description of the life of Hevelius and of his star catalogue is given by Volkoff et al. (1971) in a book celebrating the acquisition by the Brigham Young University of Hevelius' manuscript for the catalogue.

In 1679 Halley visited Hevelius and his observatory, and verified that measurements with the instruments of Hevelius, fitted with naked-eye sights, were more accurate than measurements with contemporary instruments with telescopic sights (Volkoff et al. 1971, p. 41-45). Hevelius' star catalogue was studied among others by Baily (1843), and a modern comprehensive analysis was made by Rybka (1984), who confirmed that the measurements by Hevelius were superior to those by his contemporaries.

Our study of the star catalogue by Brahe (Verbunt \& Van Gent 2010, hereafter Paper I), showed the superiority of

* Star catalogue of Hevelius is only available in electronic form at the CDS via anonymous ftp to

cdsarc.u-strasbg.fr $(130.79 .128 .5)$ or via

http://cdsweb.u-strasbg.fr/cgi-bin/qcat?J/A+A/516/A29 the modern Hipparcos Catalogue (ESA 1997) in the analysis of old star positions, due to its better completeness, accuracy and homogeneity as compared to earlier catalogues. In this paper we present a machine-readable version of the star catalogue of Hevelius, as printed in Hevelius (1690). In addition to the numbers given by Hevelius this version provides a cross-correlation with the catalogue of Brahe; identifications with stars from the Hipparcos Catalogue (ESA 1997) and on the basis of these the accuracy of the positions and magnitudes tabulated by Hevelius; and a comparison of our identifications with those of Rybka (1984).

In the following we refer to (our machine-readable version of) the catalogue of Hevelius (1690) as Hevelius, to Kepler's 1627 edition of Brahe's catalogue as Kepler, and to our emended version of this edition as KeplerE. As we will see, Hevelius also refers to the Secunda Classis, the star list that immediately follows Brahe's catalogue in Kepler (1627), and gives positions and magnitudes of those stars from the catalogue of Hipparchos/Ptolemaios that Brahe omitted from his own catalogue. Individual entries in Hevelius are numbered according to the order in which they appear, i.e. H350 is the 350th entry. A $K$ number refers to an entry in KeplerE $(K \leq 1004)$ or in Secunda Classis $(K \geq 1005)$. The sequence number within a constellation is indicated by a number following the abbreviated name of the constellation: Vul 3 is the third entry in the constellation Vulpecula in Hevelius.

\section{Description of the catalogue}

The catalogue by Hevelius is organized by constellation, and the constellations are ordered alphabetically. There are 56 constellations in the catalogue, of which 11 are new with respect 
Table 1. Constellations in the star catalogue of Hevelius.

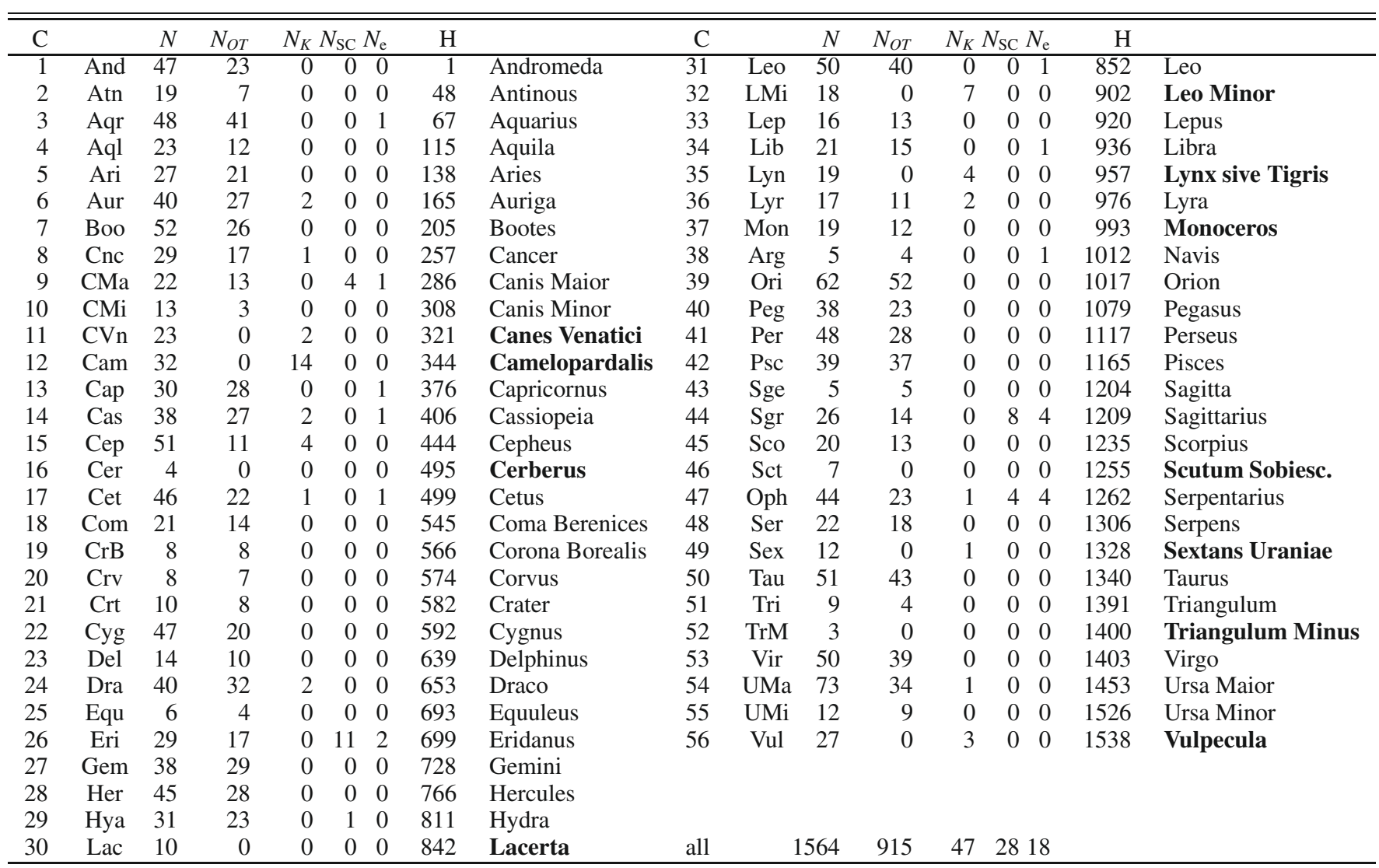

Notes. For each constellation the table gives its abbreviation, the number of stars in it, and the numbers among these of stars indicated to be in KeplerE $N_{O T}$, in KeplerE without indication $N_{K}$ and in Secunda Classis $N_{\mathrm{SC}}$, the number of empty entries $N_{\mathrm{e}}$, and the $\mathrm{H}$ sequence number of the first star in each constellation. New constallations by Hevelius - partially based on Plancius - are indicated with bold face.

to the star catalogue of Brahe (Table 1). Cerberus, Lacerta sive Stellio, Scutum Sobiescianum, Sextans Uraniae and Triangulum Minus are new constellations introduced by Hevelius. Camelopardalis and Monoceros are constellations introduced by the Dutch astronomer and cartographer Plancius for a globe by Van den Keere in 1612. Hevelius divided other constellations from Plancius, Jordanis Fluvius (River Jordan) and Tigris Fluv. / Euphrates Fluv. (Rivers Tigris and Euphrates) into Canes Venatici, Leo Minor, Lynx (sive Tigris), and Vulpecula cum Anser (see Van der Krogt 1993, p. 190-196).

The catalogue of Hevelius contains 1564 entries, including 18 empty ones for which Hevelius gives no own measurements, but only positions from other catalogues. 13 entries are repeat entries, (almost) identical to entries elsewhere in the catalogue (Table 2). Thus Hevelius gives his magnitudes and positions for 1533 independent entries.

For each entry a brief description is followed first by the sequence number of this entry in the corresponding constellation in Brahe's catalogue (Ordo Tychonis), by the magnitude given to this star by Brahe (Magnitudo Tychonis), and by the magnitude as determined by Hevelius (Magnitudo Hevelii). The magnitudes are given in integers, but are sometimes qualified by an second number 1 higher or lower than the first number (e.g. 6.7.), by the word fere (approximately), or otherwise (Table 3).

The magnitude as measured by Hevelius is followed by the position of the entry in ecliptic coordinates, given in degrees $D$, minutes $M$, seconds $S$ and sign (Gr., Min., Sec., Sig.). All numbers are integers. For the longitude, the sign is the zodiacal sign, indicated with its symbol in Hevelius' catalogue, replaced by us with an integer number $Z$ from 1 to 12 as shown in Table 2 of Paper I (from Aries $=1$ to Pisces $=12$ ). For the latitude the sign is an A or B indicating Australis (south) or Borealis (north). The longitude and latitude in decimal degrees follow as

$\lambda=(Z-1) \times 30+G+\frac{M}{60}+\frac{S}{3600}$

and

$\beta= \pm\left(G+\frac{M}{60}+\frac{S}{3600}\right)+/-$ for $\mathrm{B} / \mathrm{A}$

The equinox of the coordinates is given as Annum Christi Completum MDCLX, i.e. 1661.0.

These data are followed for each entry by the positions, where available, in ecliptic coordinates as given by Tycho, Wilhelm Landgrave of Hesse, Riccioli, Ulugh Beg, and Ptolemaios. The final information for each entry is the equatorial position as determined by Hevelius.

Of all this information our machine-readable version of Hevelius' catalogue only retains the sequence number in Brahe, and the magnitude and ecliptic position according to Hevelius.

Hevelius gives Ordo Tychonis numbers for 939 entries: 911 of these indeed are entries in KeplerE, usually but not always in the same constellation as in Hevelius; the 28 others are in the Secunda Classis of Kepler (1627). Magnitudes of stars first catalogued by Hevelius are labelled IH (i.e. Johannes Hevelius). In most cases entries which do not have a Brahe sequence number 
Table 2. Repeated entries in Hevelius.

\begin{tabular}{rrrrcl}
\hline \hline $\mathrm{H}$ & $\mathrm{H}$ & \multicolumn{1}{c}{ HIP } & $d_{1,2}\left({ }^{\prime}\right)$ & $\Delta M$ & Figure: location \\
\hline 16 & 1202 & 4463 & 1.0 & 0 & C.1: $-4.4,-16.7$ \\
321 & 1460 & 63125 & 0.0 & 0 & C. $13:-4.1,-2.0$ \\
322 & 1488 & 61317 & 0.0 & 0 & C. $13:-9.2,-1.0$ \\
349 & 689 & 59504 & 0.2 & 0 & C.14: $11.4,17.3$ \\
692 & 1536 & 73199 & 9.1 & 1 & C.55: $10.4,3.4$ \\
887 & 919 & 52457 & 0.4 & 1 & C.32: $-1.6,6.7$ \\
889 & 916 & 53417 & 0.0 & 0 & C.32: $0.3,9.2$ \\
890 & 918 & 54951 & 0.0 & 0 & C.32: $5.0,9.5$ \\
1091 & 1557 & 106140 & 0.0 & 0 & C.40: $-14.5,11.4$ \\
1145 & 1151 & 17313 & 15.7 & 1 & C.41: $0.7,-11.0$ \\
1150 & 1164 & 17886 & 0.0 & 0 & C.41: $1.9,-12.1$ \\
1154 & 1163 & 14382 & 0.0 & 0 & C.41: $0.9,12.5$ \\
1299 & 1326 & 80179 & 0.3 & 1 & C.46: $-12.7,5.7$ \\
\hline
\end{tabular}

Table 3. Magnitude qualifiers used by Hevelius.

\begin{tabular}{lcclcc}
\hline \hline$. n+1$ or $. n+1$. & + & 22 & 3.0 .6 & $\mathrm{~V}$ & $\mathrm{H} 593$ \\
$. n-1$ or $. n-1$. & - & 8 & $n$ imo major & $\mathrm{M}$ & H965 \\
fere & $\mathrm{f}$ & 9 & non nisi tub. vis. & $\mathrm{T}$ & $\mathrm{H} 956$ \\
imo $n+1$ & $\mathrm{I}$ & $\mathrm{H} 88$ & non nisi tubo visibilis & $\mathrm{T}$ & $\mathrm{H} 1250$ \\
imo $n-1$ & $\mathrm{i}$ & $\mathrm{H} 530$ & & & \\
\hline
\end{tabular}

Notes. For each qualifier for magnitude $n$ we give our indication in the machine-readable Hevelius and the number of occurrences. If it occurs only once, the $\mathrm{H}$ number is given.

have magnitudes labelled IH, but exceptions occur when the star is in another earlier catalogue, in particular the Secunda Classis of Kepler (1627) or in Riccioli (1651). Occasionally a star with a magnitude not labelled IH, thus presumably considered a previously catalogued star by Hevelius, nonetheless is without position in any of the catalogues tabulated by Hevelius. Details are given in Sect. A.1.

\section{Identification procedure}

The procedure that we follow for the identification of each star from the catalogue of Hevelius is mutatis mutandis identical to the procedure that we followed for the catalogue of Brahe, and we refer to Paper I for details. Briefly, we select all stars from the Hipparcos Catalogue with a Johnson visual magnitude brighter than 6.0, we correct their equatorial positions for proper motion between the Hipparcos epoch 1991.25 and 1661.0, then precess the resulting equatorial coordinates from the Hipparcos equinox 2000.0 to 1661.0, and finally convert the coordinates from equatorial to ecliptic, using the obliquity appropriate for 1661 .

For each entry in the Hevelius catalogue we find the nearest - in terms of angular separation - counterpart in the Hipparcos Catalogue. In general, this counterpart is selected by us as a secure identification, and given an identification flag 1 . If a much brighter star is at a marginally larger angular distance, we select that star as the secure counterpart, and give it flag 2. Especially for larger angular distances we may decide that the identification is uncertain (flag 3); and occasionally several Hipparcos stars appear to be comparably plausible as counterparts for the same entry (flag 4). An entry for which we do not find a plausible identification is flagged 5; and an entry which is identified with an Hipparcos star that already is the identification of another entry - i.e. a repeat entry - is flagged 6.
It is indicative of the high accuracy of the Hevelius catalogue that the number of problematic identifications (flags 35 ) is much smaller than in our analysis of Brahe's catalogue, notwithstanding the rather larger number of entries. In 5 cases we accept an identification with Hipparcos magnitude $V=6.1$, and in one case each with $V=6.3$ and $V=6.5$.

In thirteen cases two entries are identified with the same Hipparcos star. These are listed in Table 2 In seven pairs both stars have exactly identical coordinates, in four their coordinates differ by $\leq 1^{\prime}$. In some cases there are nearby unidentified stars, and it would be tempting to assign one of the pair to such a star, if it weren't for the too large offset required.

\subsection{Identifications by Rybka}

To compare the identifications from Rybka (1984) with those by us, we convert his identifications to an Hipparcos number. In most cases Rybka gives a Bayer or Flamsteed identification (e.g. $\alpha$ And, 7 And), or an identification from the FK4 catalogue or from the SAO catalogue. We convert these to HR numbers through the Bright Stars Catalogue (Hoffleit \& Warren 1991) and from HR via HD number to Hipparcos numbers. If this fails, we use the SIMBAD database. For H319 (=CMi 12), Rybka gives $12 \mathrm{CMi}$ as Flamsteed counterpart. We do not find a star from the Hipparcos catalogue with $V<6.5$ within $45^{\prime}$ from 12 CMi, however; our identification for H 319, HIP 39311, corresponds to the 13th star in Navis in Flamsteed's catalogue.

In some cases Rybka (1984) only provides an identification from Flamsteed's (1725) edition of Hevelius' catalogue: these look like Flamsteed numbers but have an $\mathrm{H}$ attached: e.g. $32 \mathrm{H} \mathrm{Cam}$. The stars in Flamsteed's edition are in a different order than in Hevelius, but otherwise have identical numbers (i.e. magnitudes and both equatorial and ecliptic position for 1661.0). Thus $32 \mathrm{H} \mathrm{Cam}$ is a self-reference, and does not provide an identification. In some cases we have found Hipparcos identifications for Flamsteeds Hevelius numbers via SIMBAD, but is 6 cases we have not, and we classify these as unidentified in Rybka (1984).

In 32 cases our identification suggests an emendation to Rybka's identification. These are discussed in Sect. A.2. In 21 cases this emendation leads to a different corresponding Hipparcos number, in 11 cases the correspondence is not affected.

\section{The machine-readable catalogue}

The machine-readable table Hevelius contains the following information (see Table 4). The first column gives the sequence number $\mathrm{H}$. The second column the sequence number $K$ of the corresponding star in KeplerE $(K \leq 1004)$ or in Secunda Classis $(1005 \leq K<2000)$ : these numbers are used only when Hevelius gives a Brahe sequence number $(O T)$ or an ecliptic position from Kepler, that provides an unambiguous correspondence. We give the first star in Secunda Classis the number K 1005, and continue the numbering for the following stars in order of appearance (see Verbunt \& Van Gent 2010, Paper III, in preparation; also for the exception in Sagittarius). In some cases an entry in Hevelius is identified with the same Hipparcos number as an entry in KeplerE, even though Hevelius does not indicate a Brahe sequence number or position. We consider the correspondence in these cases probable but not secure, and indicate them with $2000+\mathrm{K}$ in column two. For example, H 201 is identified with HIP 23522 as is K 11 in KeplerE; the second column in the machine-readable table Hevelius gives 2011. 
Table 4. First lines of the machine-readable table Hevelius.

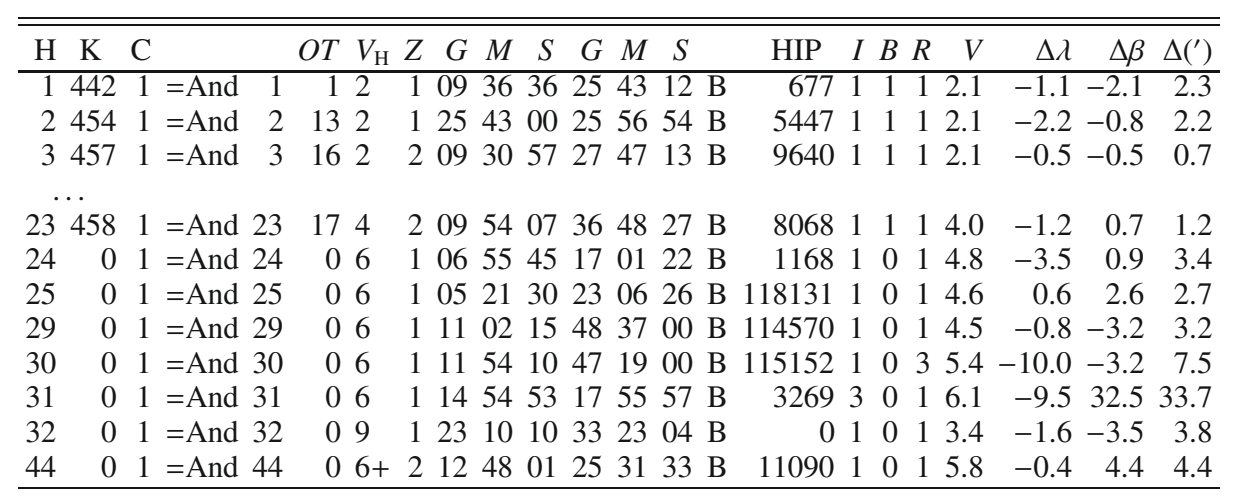

Notes. For explanation of the columns see Sect. 4.

Table 5. Frequency of flags B of identifications of corresponding entries in KeplerE as a function of our flags I.

\begin{tabular}{lrrrrr}
\hline \hline $\mathrm{I} \backslash \mathrm{B}$ & 0 & 1 & 2 & 3 & $\mathrm{All}$ \\
\hline 1 & 2 & 873 & 2 & 15 & 892 \\
2 & 0 & 5 & 0 & 0 & 5 \\
3 & 0 & 0 & 0 & 0 & 0 \\
4 & 0 & 3 & 1 & 1 & 5 \\
5 & 2 & 0 & 0 & 0 & 2 \\
6 & 0 & 1 & 0 & 0 & 1 \\
all & 4 & 882 & 3 & 16 & 905 \\
\hline
\end{tabular}

The third and fourth columns indicate the constellation: indicated with its sequence number in the catalogue and with the modern abbreviation, as listed in Table 1. For some constellations no longer in use (Antinous, (Argo) Navis, and Triangulum Minus) we introduce an abbreviation. Column 5 gives the sequence number of the entry within the constellation in Hevelius.

Columns 6-16 copy information from the original catalogue. Column 6 gives the Ordo Tychonis OT (see Sect. 2 and Sect. A.1 for details). Column 7 gives the magnitude: when the entry is indicated as non nisi tubo visibilis (not visible unless with a tube [i.e. telescope]) by Hevelius, we give it magnitude 8 ; when indicated nebulous by Hevelius, we give it magnitude 9 . Column 8 give magnitude qualifiers, as detailed in Table 3. Columns 9-12 give the ecliptic longitude, $(Z, G, M$ and $S)$ and Cols. 13-16 the ecliptic latitutde $(G, M, S$ and A/B). For this notation, see Sect. 2.

Columns 17-24 provide additional information from our analysis. Column 17 gives the Hipparcos number of our identification, and Col. 18 a flag indicating the quality of the identification, as explained in Sect. 3 (see also Table 5 in Paper I).

Column 19 flags the identification of the corresponding entry in KeplerE: a 0 if that entry is not identified, a 1 if its identification is the same as the one here in Col. 17, a 2 if its identification is to the other of a pair of possible identifications, and a 3 for a different identification. Column 20 flags the identifications by Rybka (1984), with the same notation. Column 21 gives the visual (Johnson) magnitude of the Hipparcos object given in Col. 17. Columns 22, 23 give the difference in longitude and latitude between the correct position (based on information from the Hipparcos Catalogue) and the catalogue entry. Note that the tabulation in Hevelius gives minutes and seconds, which we convert to decimal minutes $M_{\mathrm{H}}$ to compute Cols. 22 and 23. If the catalogue entry for minutes as computed from the position and proper motion in Hipparcos Catalogue is $M_{\mathrm{HIP}}$, and $M_{\mathrm{H}}$ is the
Table 6. Frequency of flags $\mathrm{R}$ of identifications by Rybka as a function of our flags I.

\begin{tabular}{|c|c|c|c|c|c|c|c|c|c|c|}
\hline \multirow[b]{2}{*}{$I \backslash R$} & \multicolumn{5}{|c|}{ in KeplerE } & \multicolumn{5}{|c|}{ Full catalogue } \\
\hline & 0 & 1 & 2 & 3 & all & 0 & 1 & 2 & 3 & all \\
\hline 1 & 5 & 880 & 3 & $\overline{4}$ & 892 & 20 & 1426 & 4 & 29 & 1479 \\
\hline 2 & 0 & 5 & 0 & 0 & 5 & 0 & 10 & 0 & 1 & 11 \\
\hline 3 & 0 & 0 & 0 & 0 & 0 & 2 & 9 & 0 & 4 & 15 \\
\hline 4 & 0 & 2 & 1 & 2 & 5 & 1 & 5 & 2 & 4 & 12 \\
\hline 5 & 0 & 0 & 0 & 2 & 2 & 7 & 0 & 0 & 9 & 16 \\
\hline 6 & 0 & 1 & 0 & 0 & 1 & 1 & 11 & 0 & 1 & 13 \\
\hline all & 5 & 888 & 4 & 8 & 905 & 31 & 1461 & 6 & 48 & 1546 \\
\hline
\end{tabular}

Notes. Left: for stars in KeplerE, right: for all entries in Hevelius.

value from the Hevelius Catalogue, then columns 22 and 23 give $M_{\mathrm{HIP}}-M_{\mathrm{H}}$. Column 24 gives the difference between correct and tabulated position in '.

\section{Analysis and discussion}

\subsection{Comparison with Brahe}

915 entries in Hevelius can be matched unambigously with an entry in KeplerE: 911 (including the repeat entry H 793) through their Ordo Tychonis, and 4 without OT through the position according to Tycho as given by Hevelius. 10 of these 914 independent entries have no position by Hevelius, among them SN 1572. Table 5 compares our identifications of the entries common to Hevelius and KeplerE: in most cases we found the same identification for both catalogues. The 16 exceptions arise because Hevelius gives a position very different from the position in KeplerE: striking examples are H 14/K 463, H 1005/K 961 and H 1099/K 441, as detailed in Sect. B.

90 entries in KeplerE, among which 12 which we were unable to identify with an Hipparcos star, have no explicit counterpart in Hevelius. The 12 unidentified stars probably have a wrong position in KeplerE, which would explain why Hevelius found no star at that position. For the others we checked whether their Hipparcos identifications occur also in Hevelius. This is the case for 57. It appears likely that Hevelius and Brahe observed the same star in these cases, but we cannot exclude an occasional chance coincidence. Many of the remaining 21 entries in KeplerE that are not matched with an entry in Hevelius have a very large position error $\Delta$ in KeplerE. The unmatched entries also include 4 stars in Argo and all 4 stars in Centaurus. 
F. Verbunt and R. H. van Gent: The star catalogue of Hevelius
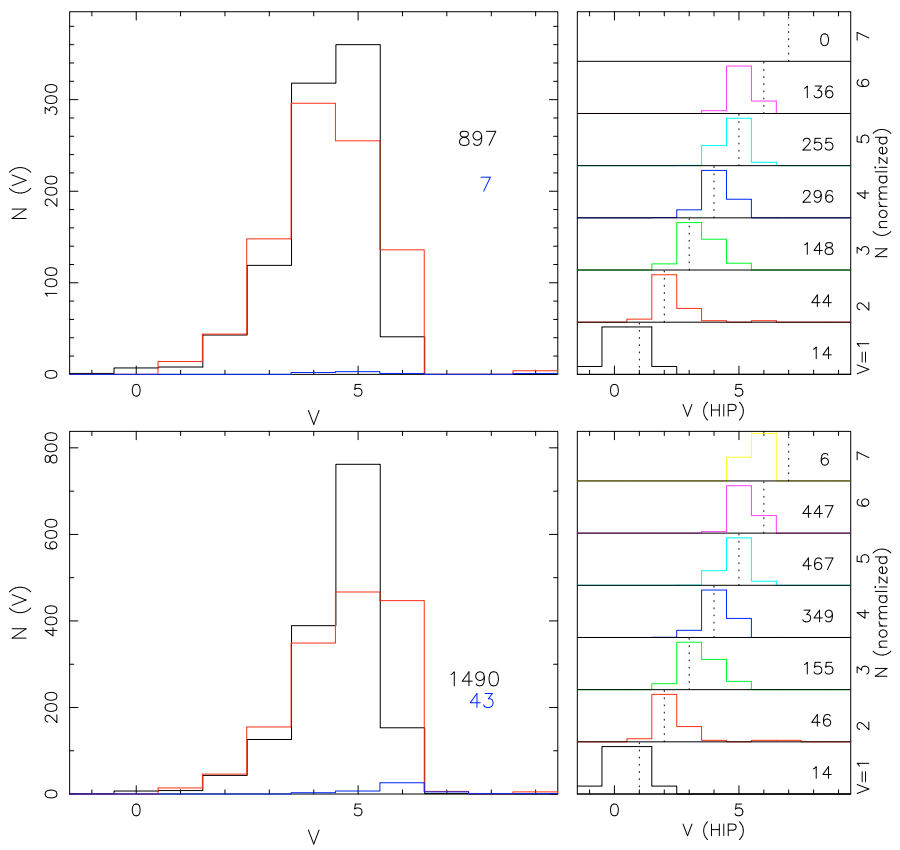

Fig. 1. Distribution of the magnitudes for all stars in Hevelius (below), and for only those stars that have a counterpart in KeplerE (above). In the large frames the histograms indicate the magnitudes according to Hevelius for stars which we have securely identified (red; flags 1-2 ) or not securely identified (blue, flags 3-5), and the magnitudes from the Hipparcos catalogue for securely identified stars (black). The numbers of securely and not-securely identified stars are indicated. The small frames give the Hipparcos magnitude distributions for securely identified stars for each magnitude according to Hevelius separately. The number of securely identified stars at each (Hevelius) magnitude is indicated.

Some remarkable features of entries in KeplerE are present in Hevelius as well. We mention in particular the three stars in Capricornus which are denoted 'nebulous' both in Hevelius and Brahe, and H 1188/K 801 which both in Kepler and Hevelius are given a B for northern latitude, whereas the correct latitude is $\mathrm{S}$ for southern (see Fig. C.44 in Paper I).

\subsection{Comparison with Rybka (1984)}

In Table 6 we compare the identifications as found by us with those given by Rybka (1984), separately for the stars in KeplerE and for all stars in Hevelius. In most cases the identifications are identical, but there are differences. We have identified 24 stars (among which 1 repeated entry) that Rybka could not identify. In 6 cases where two stars are plausible counterparts we choose the stars that Rybka did not choose. In 48 cases (among which 1 repeated entry) we do not agree with the identification in Rybka (1984); this includes 9 stars which we cannot identify. This number does not include the 21 cases where our emendation to Rybka leads to a different Hipparcos identification (see Sect. A.2). In some cases the identification given by Rybka (1984) has such a large positional offset, or is so faint, that we consider our rejection secure; in other cases we choose another closer and/or brighter star as a more plausible counterpart. Details may be found in Sect. B.
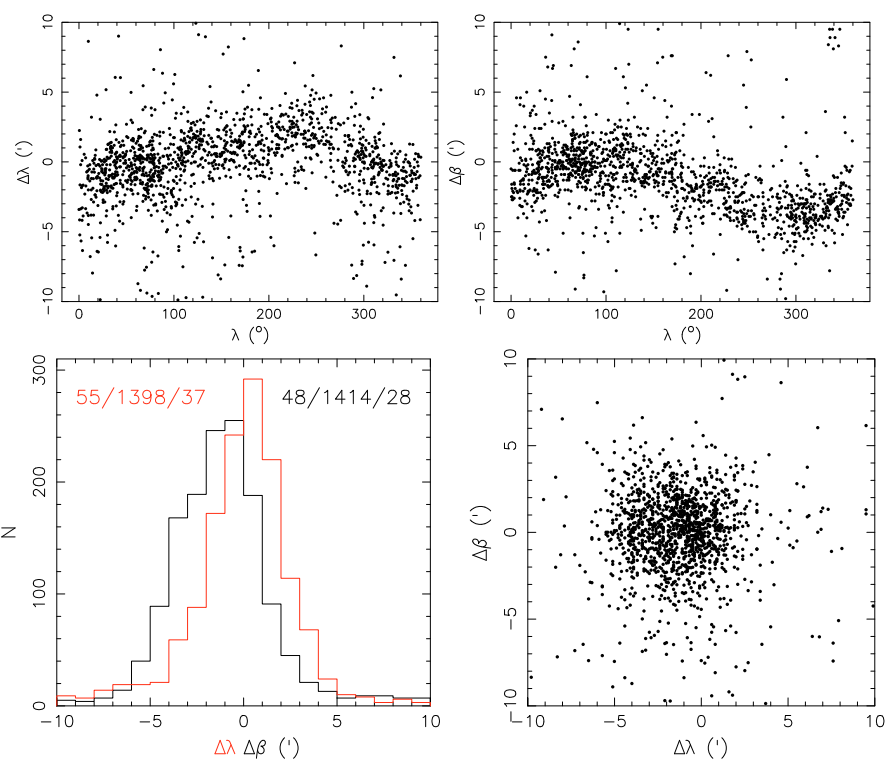

Fig. 2. Above: Correlations of the differences in longitude $\Delta \lambda \equiv\left(\lambda_{\text {HIP }}-\right.$ $\lambda) \cos \beta$ and latitude $\Delta \beta \equiv \beta_{\mathrm{HIP}}-\beta$ of the entries in Hevelius and their secure Hipparcos counterparts (converted to 1661) with longitude. Below left: distributions of $\Delta \lambda$ and $\Delta \beta$. The numbers of sources with $\Delta \lambda, \Delta \beta<$ $-10^{\prime}$, of sources included in the histogram $\left(-10^{\prime}<\Delta \lambda, \Delta \beta<10^{\prime}\right)$, and of sources with $\Delta \lambda, \Delta \beta>10^{\prime}$ are indicated. Below right: correlation between $\Delta \lambda$ and $\Delta \beta$.

\subsection{Accuracy}

Table 6 shows that there are 16 stars, i.e. one percent of the total, in Hevelius that we are not able to identify. We do not count in these the empty entries. As in the case of the Brahe catalogue, we would have to accept fainter counterparts or larger position errors to identify these; in both cases the probability of chance coincidences would increase. Other entries in Hevelius which do not have an identification in the Hipparcos Catalogue are $\mathrm{H} 32$ (= M 31), H 259 (= Praesepe) and H 1540 (= Nova Vul 1670; see Sect. B).

Figure 1 illustrates that the magnitudes assigned by Hevelius correlate well with those of their counterparts in the Hipparcos Catalogue. Only the higest magnitudes assigned by Hevelius, 6 and 7 , tend to be too high.

In Fig. 2 we show the error distributions separately for the longitudes and latitudes, as well as their correlation. The correlation distribution is roughly spherical, i.e. the errors in longitude and latitude are mostly independent. Gaussians that fit the central regions $\left(-5^{\prime},+5^{\prime}\right)$ of the distributions of $\Delta \lambda$ and $\Delta \beta$ both have widths $\sigma \simeq 2^{\prime}$; both predict fewer points at errors larger than $5^{\prime}$ than observed. The numbers of errors with absolute values larger than $10^{\prime}$ correspond to less than $10 \%$ of the number of identified entries, a similar percentage as in KeplerE. The widths of the peak of the error distributions (near $2^{\prime}$ ) and the fraction of larger errors are thus similar in Hevelius to those in KeplerE, which is an impressive achievement since the number of stars has increased by more than $50 \%$ mostly at the fainter magnitudes 5 and 6.

Figure 2 further shows that the errors in longitude $\Delta \lambda$ increase with the distance to the zero point $\Upsilon$; and that the errors in latitude $\Delta \beta$ have a roughly sinusoidal dependence on longitude. The latter dependence may be explained by an error in the value of the obliquity. Hevelius measured the obliquity in several years, and found values around $\epsilon_{\mathrm{H}}=23.506$ (Rybka 1984, p. 37); according to modern theory the obliquity in 1661 was 


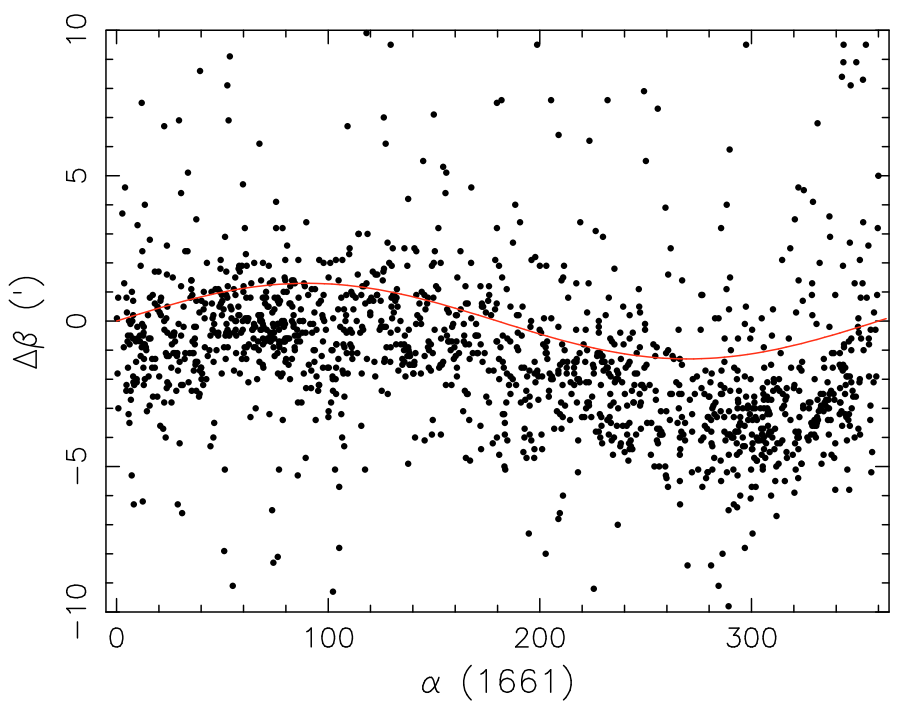

Fig. 3. Distribution of the errors in latitude in Hevelius as a function of right ascension, together with the r.h.s. of Eq. (1).
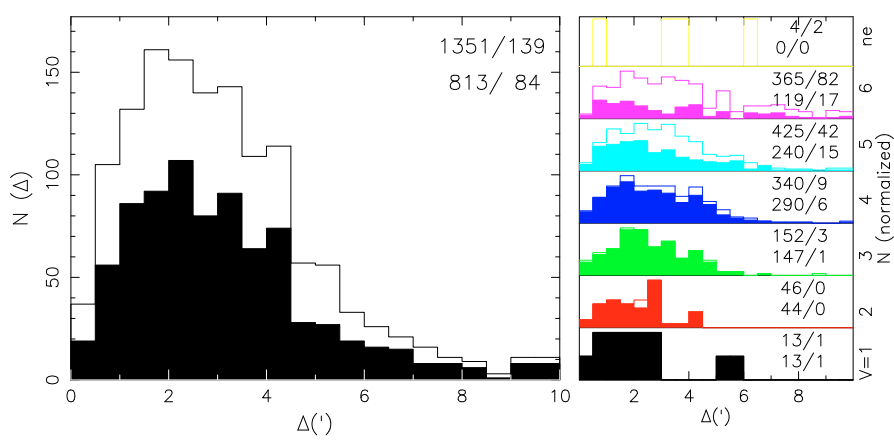

Fig. 4. Distribution of the position errors $\Delta$ for all stars in Hevelius (open histogram) and for only those stars that have a counterpart in KeplerE (solid histograms) for all securely identified stars (left) and for the securely identified stars at each Hevelius magnitude separately (right). The numbers indicate the number of stars included in the plot (i.e. with $\left.\Delta<10^{\prime}\right)$ and those excluded $\left(\Delta \geq 10^{\prime}\right)$.

$\epsilon=23^{\circ} .483$. For small declinations $\delta$, the resulting error in latitude $\Delta \beta \equiv \beta-\beta_{\mathrm{H}}$ due to the error $\Delta \epsilon \equiv \epsilon-\epsilon_{H}$ after converting equatorial to ecliptic coordinates may be written

$\cos \beta \Delta \beta \simeq-\sin \alpha \cos \epsilon \Delta \epsilon \simeq 1$ ' $3 \sin \alpha$.

The observed relation between $\Delta \beta$ and $\alpha$ is shown in Fig. 3 together with the curve $1 ! 3 \sin \alpha$ which roughly matches the phase and amplitude of the $\alpha$-dependence of $\Delta \beta$.

The average offset of longitude is virtually zero; the latitudes have an average offset of -1.4 . This average offset may be due to an underestimate by Hevelius of refraction. The distribution of the total position errors $\Delta$ in Hevelius is shown in Fig. 4. This distribution peaks roughly at the value of the width $2^{\prime}$ of the separate distributions in $\Delta \lambda, \Delta \beta$, as expected (see explanation in Paper I). The number of stars with large position errors is markedly smaller in Hevelius than in KeplerE. In particular, the number of stars with position errors larger than a degree is 21 (on a total of 1517 identified entries) in Hevelius as compared to 47 (on a total of 977 identified entries) in KeplerE. Similarly, the number of unidentified stars is 16 (of 1533 independent entries) in Hevelius and 14 (of 992 independent entries) in KeplerE. It may be concluded that the overall accuracy of the star

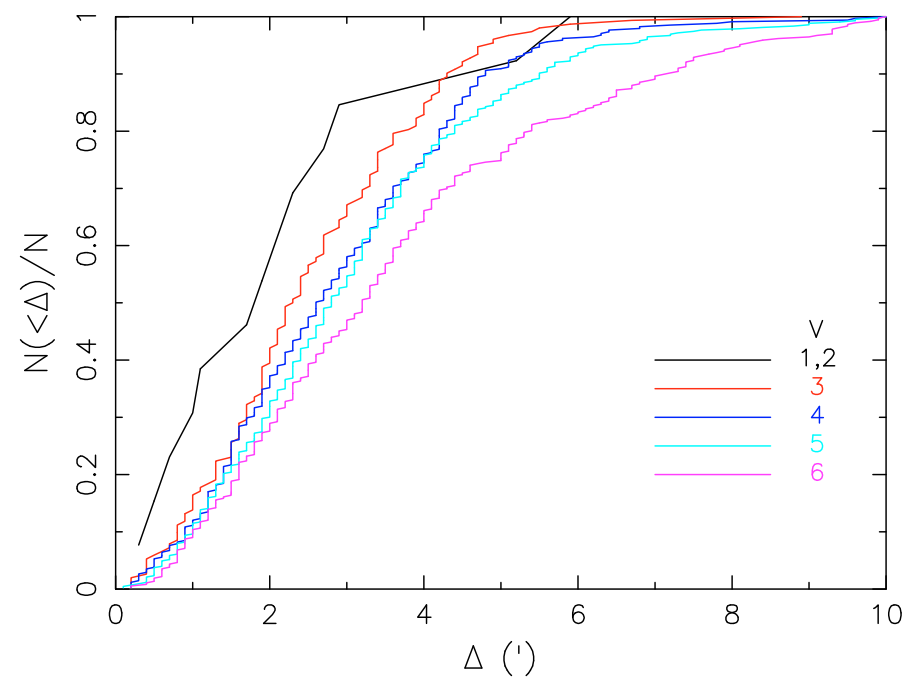

Fig. 5. Cumulative distribution of the position errors $\Delta$ in Hevelius as a function of Hevelius magnitude, showing a systematic increase in median error with magnitude.

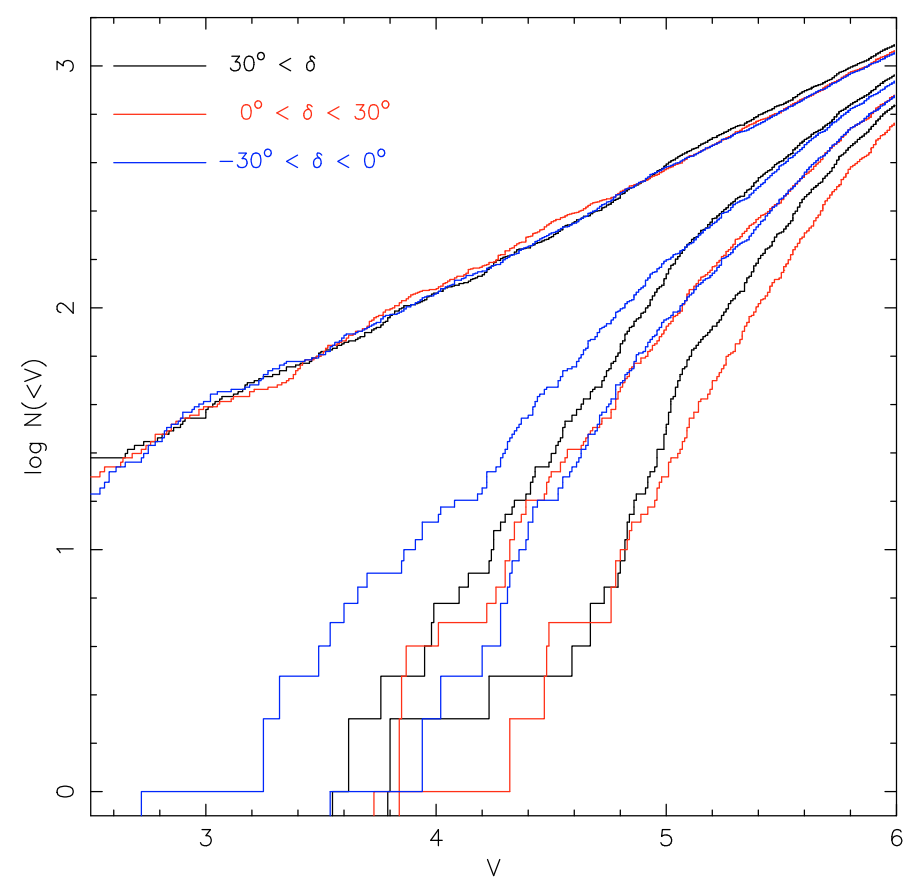

Fig. 6. Completeness of Hevelius and KeplerE as a function of magnitude and declination, as illustrated by cumulative magnitude distributions. For each range of declination (equinox 1631) the top curve shows the magnitudes for all stars in the Hipparcos Catalogue, the middle curve the Hipparcos stars not in KeplerE, and the lower curve the Hipparcos stars not in Hevelius.

catalogue of Hevelius is better than that of the star catalogue of Brahe/Kepler.

In Fig. 5 we show the cumulative error distributions for each Hevelius magnitude separately, taking magnitudes 1 and 2 together, and limiting the distributions to $\Delta<10^{\prime}$. It is seen that the median error increases slowly but systematically with magnitude. 


\subsection{New and old stars: completeness}

In Fig. 6 we investigate the completeness of Hevelius and KeplerE as function of magnitude, for three declination ranges. For this purpose we select from Hevelius and KeplerE only those entries which we have identified, and which are not repeat entries, i.e. entries with $I=1-4$. For selecting the Hipparcos stars in the latitude ranges we convert their positions to an equinox halfway between Brahe and Hevelius, viz. 1631.0. At magnitudes $V<4$ there are 348 stars from the Hipparcos Catalogue with $\delta>-30^{\circ}$, of which 5 are absent from Hevelius and 23 from KeplerE (of which 13 with $\delta<0^{\circ}$ ). At magnitudes $V<5$ there are 1138 Hipparcos stars with $\delta>-30^{\circ}$, of which Hevelius misses 141 stars (89 with $\delta<0^{\circ}$ ) and KeplerE 389 (156 with $\delta<0^{\circ}$ ). Finally, about 3500 Hipparcos stars with $V<6$ have $\delta>-30^{\circ}$, and of these some 2000 are absent from Hevelius and 2500 from KeplerE, which is just another way of saying that Hevelius and KeplerE contain about 1500 and 1000 stars visible to the naked eye, respectively. It may be noted here that the latitude of Gdansk is about 10.5 further south than that of Hven. Nonetheless, as shown in Fig. 6 KeplerE is more incomplete already at brighter magnitudes, also in the northern parts of the sky.

How many new stars did Hevelius observe? In the manuscript of the catalogue, a note dated 1681 March 31 states that 946 stars of Tycho and 617 new stars were observed (Volkoff et al. 1971, p. 72 ). This gives a total of 1563 , very close to the total of 1564 entries given in Table 1, but spuriously so since Hevelius gives no own measurements for 18 of the 1564 entries. Hevelius indicates, through an $O T$ number or a position from Tycho, for 915 entries that they are from Kepler (Table 1). For 905 of these Hevelius gives his own measurements (see Sect. 5.1). To obtain the higher number of 946 stars from the note, we have two options. One is to add the 28 stars from the Secunda Classis. (These were also measured by Brahe, according to Kepler, albeit with less accuracy). This option leaves us with too small a number. The other option is to add the 47 entries for which our identification corresponds to an identification in KeplerE. This would imply that Hevelius was aware that more stars from his catalogue corresponded to stars in Kepler than the 915 entries marked by him as such through OT or Tycho position.

If we subtract from the total number of 1564 entries in Hevelius all 962 that have a counterpart in KeplerE and further subtract the 28 entries that have a counterpart in Secunda Classis, we find a number of 574 entries first measured by Hevelius. 573 of these are stars, the other one is M 31.

Cerberus, Lacerta, Scutum, Sextans and Triangulum Minus, the truly new constellations by Hevelius, contain a total of 36 stars, of which only one possibly corresponds to a star in KeplerE (H1328 / K959: the position error of K959 is 2.5 , so a chance coincidence is possible). Monoceros and Camelopardalis, two constellations retained by Hevelius from Plancius, contain twelve and up to fourteen stars from KeplerE, respectively (Table 1). The four constellations fashioned by Hevelius from two constellations by Plancius contain up to sixteen stars from KeplerE. We use the qualification "up to" for the stars listed under $N_{K}$ in Table 1 because some of the correspondences between Hevelius and KeplerE may be chance coincidences. The six constellations retained of refashioned from Plancius by Hevelius contain 138 stars, so even accepting all 42 correspondences as real, we still find that a large majority of stars in these constellations was first observed by Hevelius.
Acknowledgements. This research has made use of the SIMBAD database, operated at CDS, Strasbourg, France. We thank Dr. Jarosław Włodarczyk for providing us with a copy of Rybka (1984), and Oliwia Madej for help in reading parts of it. This research is supported by the Netherlands Organisation for Scientific Research under grant 614.000.425.

\section{References}

Baily, F. 1843, MRAS, 13, 1

Brahe, T. 1598, Stellarum octavi orbis inerrantium accurata restitutio (manuscript), edited by Dreyer 1916, p. 333

Brahe, T. 1602, Astronomiae Instauratae Progymnasmata, edited by Dreyer 1915 , p. 258

Dreyer, J. L. E. 1915, Tychonis Brahe Dani Scripta Astronomica, Vol.II (Gyldendal, Kopenhagen)

Dreyer, J. L. E. 1916, Tychonis Brahe Dani Opera Omnia, Vol.III (Gyldendal, Kopenhagen)

ESA 1997, The Hipparcos and Tycho Catalogues (SP-1200, ESA Publications Division, Noordwijk)

Flamsteed, J. 1725, Historia Coelestis Brittanicae, Volumen Tertium (Meere, London)

Hevelius, J. 1690, Catalogus Stellarum Fixarum (Johann Stolle, Gdansk)

Kepler, J. 1627, Tabulae Rudolphinae (Jonas Saur, Ulm)

van der Krogt, P. 1993, Globi Neerlandici: the production of globes in the Low Countries (HES, Utrecht)

Rawlins, D. 1993, DIO 3, 1

Riccioli, G. B. 1651, Almagestum novum (Victorius Benatius, Bologna)

Rybka, P. 1984, Katalog Gwiazdowy Heweliusza (Polska Akademia Nauk, Wroclaw)

Shara, M. M., Moffat, A. F. J., \& Webbink, R. F. 1985, ApJ, 294, 271

Verbunt, F., \& van Gent, R. H. 2010, A\&A, 516, A28 (Paper I)

Volkoff, I., Franzgrote, E., \& Larsen, A.D. 1971 Johanes Hevelius and his catalog of stars (Brigham Young University Press, Provo, Utah)

\section{Appendix A: Annotations and emendations}

\section{A.1. Annotations to Hevelius}

In 28 cases the Ordo Tychonis given in Hevelius is larger than the number of stars in the corresponding constellation in KeplerE, and the OT actually refers to a star in Secundis Classis. Stars in Secunda Classis are usually given OTs that follow by continuation of the numbering from the last star in the corresponding constellation in KeplerE. We identify entries from Hevelius with stars from KeplerE or Secunda Classis when the positions given by Hevelius as due to Tycho have a longitude higher by $51^{\prime}$ (the correction applied by Hevelius for precession from 1601 to 1661) and the same latitude as in Brahe's catalogue, accepting round-off errors of 0.5 in $\mathrm{M}$ (minute) for each coordinate. Such is the case for: H 296, H 298, H 299, H 302 (CMa); H 716 - H 726 (Eri); H 834 (Hya); H 1218 - H 1221, H 1229 - H 1232 (Sgr); H 1275 - H 1278 (Oph). For two stars in the Pleiades, see below.

In 21 cases the Ordo Tychonis given in Hevelius refers to a different constellation in KeplerE. These are H 258 (Cnc, OT refers to Hya); H 273 (Cnc, OT refers to CMi); H 993 - H 1002 (Mon, OTs refer to Hya); H1202 (Psc, OT refers to And); H 1241 - H 1243 (Sco, OTs refer to Lib); H1314, H 1317 H 1320 (Ser, OTs refer to Oph).

In 9 cases a position from Tycho is given by Hevelius, but no OT number. In 4 cases the star is in KeplerE, viz. H 415 (Cas, SN 1572) = K 267; H 521 (Cet) = K 919 (Eri); H 1004, H 1005 (Mon) = K 958, K 961 (Arg; with an emendation, see Sect. B) and in 5 cases the star is in Secunda Classis: H 777 (Her, SC: Oph); H 1003 (Mon, SC:Hya); H 1213, H 1216 (Sgr, SC: Sgr); H 1289 (Oph, SC: Oph). In one case, H 1016 (in Arg), a Tycho position by Hevelius $(\lambda=\Omega 0151,4900 \mathrm{~A})$ does not match a position either in KeplerE, or in Secunda Classis, although it is 


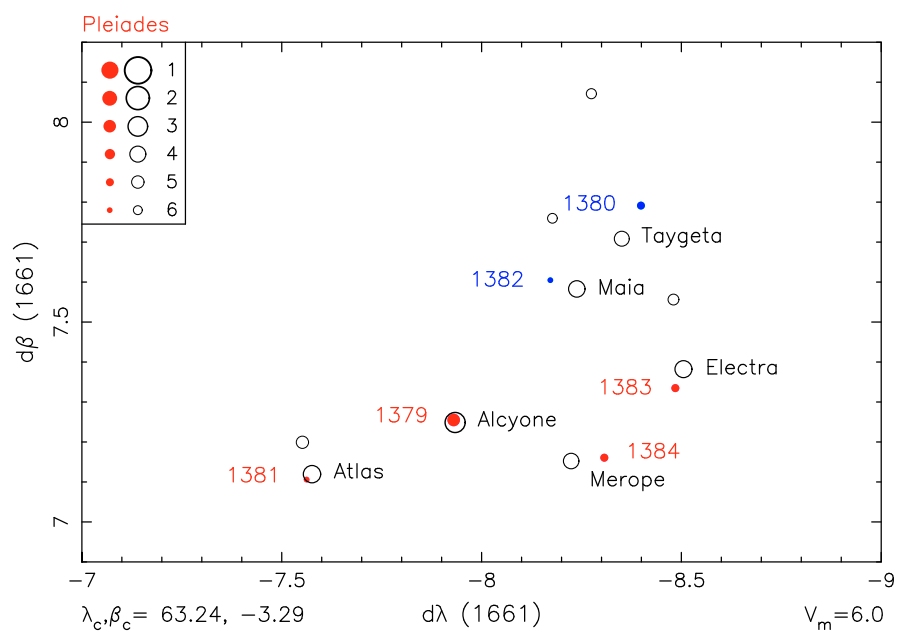

Fig. A.1. Pleiades. In red $\mathrm{H}$ numbers of stars also present in KeplerE, in blue stars added by Hevelius.

close to two stars in Argo in Secunda Classis (viz. K 1167 and K 1168).

In 5 cases a magnitude from Tycho is given by Hevelius, but no OT number or Kepler position: H 321, H 322 (in RS CVn, see Table 2; H 906, H 909, H910 (in LMi)

In 15 cases Hevelius does not mark the magnitude IH, thereby indicating that the star has been previously catalogued, but does not give a Tycho magnitude or position. These are H 108 (Aqr), H 520 (Cet; position from Riccioli), H 611 and H 613 - H 617 (Cyg), H 835 (Hya), H 911, H 913, H 914, H 916, H 918, H 919 (in LMi), H 1467, H 1468 (UMa).

Hevelius lists six stars in the Pleiades (see Fig. A.1). These correspond to entries in KeplerE for those four stars for which OTs are given, and to entries in Secunda Classis for the two other stars. If we analyse the positions as measured by Hevelius, we find that the entries from KeplerE that we identified with Electra and Merope are coupled in Hevelius to positions that match Maia and Electra. We have emended these, as listed below where the first Taurus sequence number is our emended version and the second the OT as given by Hevelius.

\begin{tabular}{lllll}
\hline \hline Alcyone & H 1379 & HIP 17702 & Tau 32 & 32 \\
Taygeta & H 1380 & HIP 17531 & - & 30 \\
Atlas & H 1381 & HIP 17847 & Tau 33 & 33 \\
Maia & H 1382 & HIP 17573 & - & 0 \\
Electra & H 1383 & HIP 17499 & Tau 30 & 31 \\
Merope & H 1384 & HIP 17608 & Tau 31 & 0 \\
\hline
\end{tabular}

\section{A.2. Emendations to Rybka}

For each emendation we list the $\mathrm{H}$ sequence number, the original text in Rybka (1984), our emendation, and a 1 (one) if the emendation changes the identification, or is required to obtain a unique identification. When Rybka gives two names for one identification, one of which corresponds to our identification, and the other must be emended to do so, we do not give a 1 .

\begin{tabular}{|c|c|c|c|c|c|c|}
\hline $\mathrm{H}$ & Rybka & emend. & & $\mathrm{H}$ & Rybka & emend. \\
\hline 55 & $v \mathrm{Aql}$ & $v \mathrm{Aql}$ & 1 & 602 & $\iota \mathrm{Cyg}$ & $\iota^{2}$ Cyg 1 \\
\hline 64 & $1 \mathrm{Psc}$ & $1 \mathrm{Aqr}$ & 1 & 671 & 63 Dra & 64 Dra \\
\hline 66 & 5 Psc & $5 \mathrm{Aqr}$ & 1 & 678 & $\psi$ Dra & $\psi^{1}$ Dra 1 \\
\hline 86 & $\tau \mathrm{Aqr}$ & $\tau^{2} \mathrm{Aqr}$ & & 733 & $6 \mathrm{Gem}$ & $\sigma$ Gem 1 \\
\hline 148 & $\rho$ Ari & $\rho^{3}$ Ari & 1 & 828 & $\phi$ Hya & $\phi^{3} \mathrm{Hya}$ \\
\hline 212 & $\kappa$ Boo & $\kappa^{2}$ Boo & & 926 & $v$ Lep & $v$ Lep 1 \\
\hline 228 & $\xi \mathrm{CrB}$ & $\zeta^{1} \mathrm{CrB}$ & 1 & 942 & $\zeta \mathrm{Lib}$ & $\zeta^{4} \mathrm{Lib} 1$ \\
\hline 265 & $\mu \mathrm{Cnc}$ & $\mu^{2} \mathrm{Cnc}$ & & 1003 & 30 Нуа & 30 Mon \\
\hline 284 & $\pi \mathrm{Cnc}$ & $\pi^{2} \mathrm{Cnc}$ & 1 & 1004 & 31 Hya & 31 Mon1 \\
\hline 348 & UZ Cam & VZCam & & 1047 & $\psi$ Ori & $\psi^{2}$ Ori 1 \\
\hline 403 & $\xi \mathrm{Cap}$ & $\xi^{2} \mathrm{Cap}$ & 1 & 1084 & $\chi$ Peg & $\gamma$ Peg 1 \\
\hline 460 & 16 Cep & 24 Cep & 1 & 1094 & $\pi \mathrm{Peg}$ & $\pi^{2}$ Peg \\
\hline 461 & 24 Cep & 16 Cep & 1 & 1196 & $v$ Psc & $v$ Psc \\
\hline 466 & $7 \mathrm{Cas}$ & $1 \mathrm{Cas}^{2}$ & 1 & 1227 & $81 \mathrm{Sgr}$ & $61 \mathrm{Sgr}$ \\
\hline 538 & 32 Eri & 22 Eri & 1 & 1367 & $\omega \mathrm{Tau}$ & $\omega^{2}$ Tau 1 \\
\hline 559 & $2 \mathrm{Com}$ & $2 \mathrm{Boo}$ & 1 & 1372 & $\sigma$ Tau & $\sigma^{2} \mathrm{Tau}$ \\
\hline
\end{tabular}

\section{Appendix B: Notes on individual identifications}

H 14, near $-14,12$ in Fig. C.1, corresponds to K 463, but in Hevelius is much closer to the other stars in Andromeda, i.e. much further south, than in KeplerE.

H 16 is identified with 65 Psc (=HIP 3885) by Rybka, but this star is at 4.5 .

H 30 is identified by Rybka with HIP 115022, brighter but further $(V=4.8, d=17$ ! 2$)$.

H 32 is the Andromeda Nebula M 31.

H 98 is marginally closer to HIP $117089(V=5.2, d=31.5)$, but we agree with Rybka that the brighter HIP 116901 is the counterpart, given the relative offsets of other stars in the area to their counterparts (Figs. C.3, C.4).

$\mathrm{H} 99$, about equally distant from $\mathrm{H} 98$ and $\mathrm{H} 100$ in Hevelius, is identified with HIP 117218 (Fig. C.4); its counterpart in KeplerE lies closer to the counterpart of $\mathrm{H} 98$ and is therefore identified with HIP 117089.

H 174 near $+3,+3$ in Fig. C.7, is close to $\beta$ Aur (= HIP 28360) in Hevelius, i.e. much further south than its counterpart K 310 in KeplerE.

H 185. Its counterpart in KeplerE lies between HIP 25541 and HIP 24879 and is identified with the latter (Fig. C.8).

H 196, H 197 and H 198 are identified by Rybka with HIP 24727 , HIP 24504 and unidentified respectively. Fig. C.8 shows that our identifications HIP 24799, HIP 24727 and HIP 24504 are better matches. Alternatives to our identifications for H 196 and H 198 are HIP 24879 and HIP 25291, respectively.

H 200, near $-5,-13$ in Fig. C.7, is identified by Rybka with HIP 25492 (near -4,-12). Our identification is a better positional match.

H 234, near 5.6,-16.8 in Fig. C.9, is identified by Rybka with HIP70738 ( $V=5.9, d=16$ '.2); our identification is brighter and nearer.

H 282-283, near $-2,+6$ and $+1.3,+3.6$ in Fig. C.10, only have distant counterparts in the Hipparcos Catalogue with $V \leq 6.0$. Rybka gives fainter counterparts for each, viz. HIP $41404(V=$ $\left.6.3, d=1^{\prime}\right)$ and HIP $41940\left(V=6.4, d=53^{\prime}\right)$.

H 296, near $-0.4,1.3$ in Fig. C.11, is "difficult to identify" according to Rybka, but clear with Hipparcos data.

H 306, near 5.5,-2.8 in Fig. C.11, is identified by Rybka with HIP 34981 near 4.6,-4.3.

H313, near $-2.4,-2.4$ in Fig. C.12, is identified by Rybka with HIP 36723, slightly brighter but further $(V=5.6, d=14$.9 $)$ than our identification. 
H314, near 2.4,0.9 in Fig. C.12, is identified with Rybka with HIP 39213, indeed the nearest star $(3.9,0.5)$ but in our view too far, at 1.5 .

$\mathrm{H} 321$ is the combined light of $\alpha^{1} \mathrm{CVn}$ (HIP 63121, $V=5.6$ ) and $\alpha^{2}$ CVn (HIP $63125 V=2.9$ ), separated by $19^{\prime \prime}$.

$\mathrm{H} 326$, is identified by us with the first of two possibilities given by Rybka.

H 329, near 1.2,-2.5 in Fig. C.13, is identified by Rybka with HIP 64927 (near 1.9,-3.5), slightly brighter but much further than our identification.

H331, near $-1.8,1.4$ in Fig. C.13, is identified by Rybka with HIP 64692 (near -2.0,2.1), fainter and further than our identification.

H338, is identified by us with the second of two possibilities given by Rybka.

H 346, near 4.1,15.1 in Fig. C.14, is identified by Rybka with HIP 49688, both fainter and further $\left(V=6.3, d=42^{\prime}\right)$ than our identification.

H 376, near $-10,+7.6$ in Fig.C.15, is closer to HIP 100027 ( $\alpha^{1}$ Cap, $d=4.6$ ) than to HIP $100064\left(\alpha^{2}\right.$ Cap, $d=5$ 5.1) but should still be identified with the latter. $\mathrm{H} 404$ is the counterpart of HIP 100027, described by Hevelius as In cornu borealis duarum contiguarum occidentalis (In the northern horn of the two adjacent ones the western one).

H400 Non videtur amplius (Is no longer seen) according to Hevelius, which is understandable as Brahe's position was erroneous (Rawlins 1993, see also Paper I).

H 405, near 8.8,0.5 in Fig.C.15, is identified by Rybka with HIP 107517 fainter and much further $\left(V=5.6, d=3^{\circ}\right)$ than our identification. Rybka gives HIP 107517 also as the identification of H399, where we agree.

H 429-432, four stars near 6,7 in Fig. C.16, had wrong positions in Brahe's catalogue and were assigned other identifications, even though this left relatively bright stars in Cassiopeia unidentified (see notes with K 236-238 and Fig. C.14 in Paper I; and Rawlins 1993, D 585-588). Hevelius decided to identify these entries in Brahe with the brighter stars.

H 436, near $-5.1,4.5$ in Fig. C.16 is identified by Rybka with HIP 2876 near $-6.1,3.2$, but this star is too far to be a reliable counterpart; we leave this star unidentified.

$\mathrm{H} 438$, near $-0.2,2.2$ in Fig. C.16 is identified by Rybka with the the Mira variable RV Cas, but this star is almost $17^{\circ}$ from the position of $\mathrm{H} 438$.

H 462, near 11.4,-2.5 in Fig. C.17, is identified by Rybka with HIP 1296 , almost $3^{\circ}$ away.

$\mathrm{H}$ 463. We list the same counterpart as Rybka; an alternative is HIP $108165, V=5.7, d=32$ '.7.

H 467-468, near $-12.9,16.1$ and $-12.1,15.7$ in Fig. C.17 are identified by Rybka with HIP 100097 and HIP 101084, near $-15.6,13.0$ and $-15.0,11.5$.

H 492, near 17.6,11.8 in Fig C.17, is identified by Rybka with HIP 84535, but this star is faint and rather far $(V=6.3, d=$ $16.5)$.

H 523, near 5.5,1.0 in Fig. C.19, is described as Nova in collo Ceti (New star in the neck of the Whale) by Hevelius, and is Mira Ceti $(o$ Cet). It is listed in the Hipparcos catalogue at $V=6.5$ (sc. average) but its magnitude at maximum is close to 2 .

H 525 is erroneously given the same identification by Rybka as H 524 .

H 557, near $-0.4,3.2$ in Fig. C.20, is identified by Rybka with HIP 62886, near $-0.9,-1.2$, but we identify H 562 with this star. We consider H 557 unidentified, just as its corresponding entry K 483 in Brahe's catalogue.
H 562, near $-0.9,-1.2$ in Fig. C.20, is identified by Rybka with HIP $6424=\alpha$ Com, near $4.3,-2.8$, clearly not compatible with its position.

H 570, near 3.2,1.9 in Fig. C.21, is identified by Rybka with $\rho \mathrm{CrB}=\operatorname{HIP} 78459\left(V=5.4, d=208^{\prime}\right)$. Our identification corresponds to $\iota \mathrm{CrB}$.

H 581. HIP 61910 corresponds to the combined flux of the two stars given by Rybka, SAO157447/8.

H593 is PCygni. Hevelius gives the magnitude as 3.0.6 which we interpret as indicating a range of 3 to 6 , in agreement with the actual variations of this star. Rybka does not identify this entry. H604. An alternative identification is HIP 99639, closer but a magnitude fainter $(V=4.8, d=2$ '.9).

H619, near 9.3,10.8 in Fig. C.24. The nearest Hipparcos star with $V \leq 6.0$ is HIP $103956\left(V=5.9, d=81^{\prime}\right.$; Rybka identifies with HIP $103530 V=5.6, d=4^{\circ}$. We consider this entry unidentified.

H 636, near -9.6,3.0 in Fig. C.24. HIP 97367 is closer to this entry but much fainter $(V=5.8, d=41$ '.7) than our identification. H649, near 1.0,1.7 in Fig. C.25, would be a good match to HIP 103294 if a correction of $2^{\circ}$ is made to the longitude. The counterpart given by Rybka is too faint and too far $(V=6.3$, $d \simeq 5^{\circ}$.

H 678, H 679. H 678 corresponds to the close pair HIP 86614, HIP 86620. The entry K94 in the Brahe Star Catalogue corresponding to $\mathrm{H} 679$, had a position closer to HIP 87728, but a description which better matches HIP 89937. The position in Hevelius also matches HIP 89937. See Fig. C.5 in Paper I.

H 690, near 23.1,26.3 in Fig. C.26 is identified by Rybka with $\operatorname{HIP} 61281(d \simeq 5.5)$.

H 698 ( $\epsilon$ Equ) corresponds to the combined flux of HIP 103571 and HIP 103569 (separated by $10^{\prime \prime}$ ).

H 714, near -11.6,9.7 in Fig. C.28, lies between HIP 14293 and HIP 14168, somewhat closer to the latter. The corresponding entry in Brahe's star catalogue K912 is closer to HIP 14293. The counterpart given by Rybka, HIP 16989, is almost $9^{\circ}$ off.

H 737, near 4.8,2.0 in Fig. C.29, lies between HIP 36429 and HIP 36393, somewhat closer to the latter. The corresponding entry in Brahe's star catalogue K 556 is closer to HIP 36429. Rybka mentions both as possible counterparts.

H 790, near -16.7,8.6 in Fig. C.30, lies between HIP 75793 (= $v^{1}$ Boo) and HIP $76041\left(=v^{2}\right.$ Boo), somewhat closer to the latter. Rybka identifies this entry with HIP 75793.

H 792, near 3.8,19.3 in Fig. C.30, is identified by Rybka with HIP $86173(V=7.3, d=4$.9). Our identification is brighter and closer.

H 794, near 5.9,19.5 in Fig. C.30, is identified with HIP 87280 by Rybka. Although variable, this star is too faint and too far ( $V=6.8, d=26^{\prime}$.4) to be a reliable counterpart. We prefer to leave this star unidentified, as its correpondent entry K 175 in Brahe's star catalogue (Paper I).

H 831, near 4.3,-9.6 in Fig. C.57, is between HIP 54255, with which we identify it, and HIP 54204, slightly further but brighter $(V=4.9, d=5$ '.6), with which Rybka identifies it.

H 837, near -27.6,-6.1 in Fig. C.57, lies between HIP 44961, with which we identify it, and HIP 44833, with which Rybka identifies it, even though it is both fainter and further $(V=5.6$, $d=12 ! 2$ ).

H 838, near 32.0,4.3 in Fig. C.57 is identified with the Mira variable R Hya by Rybka. Our identification procedure did not find this possibility, due to the faint average magnitude $(V=6.4)$, but the star is highly variable and we agree with Rybka that this is a plausible identification. 
H 849, near 0.1,-4.3 in Fig. C.31, is the combined light of HIP 111544 and HIP 111546 (separation 22'.3)

H 887, near -1.6,6.7 in Fig. C.32, has a position in Hevelius very different from its position in Brahe, where it lies above HIP 52422 (near -2.9,9.5)

H 895, near $-3.9,-4.5$ in Fig. C.32 is identified by Rybka with HIP 50755, fainter and far from the correct position $(V=6.2$, $\left.d=104^{\prime}\right)$.

H 905, near $-12.2,-1.4$ in Fig. C.33, lies between HIP 46652 $\left(V=5.9, d=44^{\prime} .1\right)$ and HIP $46735(V=5.4, d=50.5)$. Rybka identifies H 905 with the nearer star, we think either star is possible.

H 913, near 4.1,4.0 in Fig. C.33, is near three stars, HIP 53426 $\left.(V=5.0), d=36^{\prime}\right)$, HIP $53377\left(V=5.7, d=40^{\prime}\right)$ and HIP $53229\left(V=3.8, d=44^{\prime}\right)$. We agree with Rybka in considering the brightest star to be the more plausible counterpart. H 936 is closer to HIP $72603\left(\alpha^{1} \mathrm{Lib}, V=5.2, d=3 ! 3\right.$ ) than to HIP $72622\left(\alpha^{2}\right.$ Lib), but the latter is much brighter and therefore the counterpart. H 956 is the counterpart of $\alpha^{1} \mathrm{Lib}$, and Hevelius notes non nisi Tub. vis. (not visible without tube, i.e. telescope). H 954, H 955, near 3.0,-4.5 in Fig. C.34, are identified by Rybka with HIP $76880\left(d=125^{\prime}\right)$ and HIP $76628\left(d=88^{\prime}\right)$ respectively, but both are much closer to HIP 76106. We identify H 955 as the closest match to HIP 76106, and leave H 954 unidentified. H 956: see H 936.

H 979, near $-1.2,0.5$ in Fig. C.37, is identified by us with HIP 91919, but HIP91926 is at a comparable distance and marginally brighter $(V=4.6, d=4$.'6). Rybka considers H 979 to be the combined light of the two Hipparcos stars that are separated by 3.5 .

H 980, near $-1.5,-1.5$ in Fig. C.37, is the combined light of HIP 91971 and HIP 91973 (separated by 44").

H 981, near 0.3,-2.5 in Fig. C.37, lies between HIP 92791 and the fainter HIP 92728, closer to the brighter star. Rybka lists both stars as possible counterparts.

H 986, near 0.6,-6.9 in Fig. C.37, lies closest to HIP 93193, with which H9978 is identified, and corresponds to the fainter star to the south, HIP93279. In the star catalogue of Brahe its position is closer to that counterpart.

H 1005 (Mon 13) has no OT number, but Hevelius gives a Brahe position which after subtraction of his $51^{\prime}$ correction for precession, exactly matches the longitude and latitude of K 961 (Arg 11) in KeplerE, if one accepts that Hevelius' value $Z=4$ is an emendation of Brahe's $Z=6$. In his edition of Brahe's catalogue Rawlins (1993) makes exactly this emendation, which leads to a very good match of K 961 with HIP 37447 ( $\alpha$ Mon).

H 1027 is identified by us with HIP $26268(d=2$ '.7) and by Rybka with HIP 26237 ( $d=6 ! 4$ ). The corresponding entry in Brahe's star catalogue, K 870, is closer to HIP 26237: see Fig. C.48 in Paper I.

H 1063-5. Hevelius lists H 1064 as two magnitudes fainter than $\mathrm{H} 1063$ and $\mathrm{H} 1065$. This, and the close match in the positions of H 1063 and H 1965, near 5.6,15.2 and 7.8,15.0 in Fig. C.39, with HIP $27913\left(=\chi^{1}\right.$ Ori) and HIP $28716\left(=\chi^{2}\right.$ Ori $)$, respectively, excludes the interpretation of Rybka, who identifies H 1064 with $\chi^{2}$ Ori and H 1065 with HIP 29650 (near 10.3,13.9)

$\mathrm{H} 1073$, near $4.7,-14.3$ in Fig. C.39, is closer to HIP 28325 which however is identified with $\mathrm{H} 1075$. We agree with Rybka that this leaves HIP 27713, near 3.8,-13.8 as a plausible identification for $\mathrm{H} 1073$.

H 1099, near 8.6,-0.6 in Fig. C.40, has a very different position in Hevelius from it entry K 441 in KeplerE, which we identified with HIP 112997 (near -0.9,-3.6).
H 1127, near $-4.8,15.2$ in Fig. C.41, is identified by us with HIP 10729 just north of it. The corresponding entry in KeplerE $\mathrm{K} 268$ has a position closer to HIP 11060 (near -5.0,14.1) and is identified accordingly. Rybka identifies H 1127 with HIP 11060. H 1148, near -7.4,9.0 in Fig. C.41, lies closest to HIP 11220 $(V=5.2, d=11.5)$, but we agree with Rybka that HIP 11313 is the more likely counterpart

H 1151, near 0.7,-11.0 in Fig. C.41, is identified with the same star as $\mathrm{H} 1145$, and thus a repeat entry

H 1159, near 9.2,-12.2 in Fig. C.41, lies between HIP 20579 and HIP 20591, slightly closer to the former, with which we identify it. Rybka mentions both as possible counterparts.

H 1185, near 17.4,2.7 in Fig. C.42, lies between HIP 6732, which we take as the counterpart, and the slightly brighter and slightly more distant HIP $6706(V=5.4, d=4.2)$.

H 1188, near 18.2,-5.0 in Fig. C.42, lies closest to HIP 7535 as did its counterpart in Kepler; in KeplerE we emended its latitude sign from B to A which gives an excellent match with HIP 8198 (near 18.2,-8.3). Remarkably, Hevelius also gives a northern latitude.

H 1191, near 13.7,12.6 in Fig. C.42, is identified with a $V=6.1$ star, as was its counterpart K 808 in KeplerE.

H 1226, near 8.5,6.6 in Fig. C.44, is identified by us with HIP 97063. Rybka identifies H 1226 with HIP 96950 (near 8.7,5.8) which is our identification of the corresponding entry K 709 in KeplerE, which had a position closer to HIP 96950.

H 1229, near 10.8,-5.9 in Fig. C.44, is closest to HIP 98688, which however is the counterpart of H 1232. Rybka identifies H 1229 with HIP 98066 (near 9.9,-4.8, V= 4.7) but HIP 98162 (near 10.0,-5.7) is closer and slightly brighter.

$\mathrm{H} 1234$, near $-2.3,1.5$ in Fig. C.44, is identified by Rybka with the pair $v^{1}-v^{2} \operatorname{Sgr}$ (HIP 92761 and HIP 92845, near -3.3,0.8). We think this is plausible, but not certain. $v^{1}-v^{2} \mathrm{Sgr}$ are separated by 13.8; HIP 92845 is closest to H 1234.

H 1248, near 7.3,-1.4 in Fig. C.45, is identified by Rybka with HIP 80079 (near 6.4,-2.4), rather further from H 1248 than our identification.

H 1250 is located very close to H 1245 by Hevelius: Prope Supr. Front. Duar. Austr. Infer. adhaeret alteri, non nisi Tubo visibilis: ex occultatione stabilita. H 1245 is Hip $78933\left(\omega^{1} \mathrm{Sco}\right)$, and it would be tempting to identify H 1250 with HIP 78990 ( $\omega^{2}$ Sco). As Rybka remarks, two stars separated by 14.6 do not fit this description.

$\mathrm{H} 1280$ and $\mathrm{H} 1281$ have positions rather different from the corresponding entries in KeplerE, K 354 and K 355 . In KeplerE these stars have very uncertain identifications (see Fig. C.20 of Paper I), but the more accurate positions in Hevelius lead to unambiguous identifications.

H 1293 corresponds to the combined light of HIP 81634 and HIP 81641 , separated by 1'.1 and thus not separable by the naked eye.

H 1296, near 0.4,16.5 in Fig. C.48, is identified by Rybka with HIP 84626, near 3.2,-17.9, clearly not correct.

H 1337 is identified by Rybka with HIP 51592, indeed closer than our suggested identification, but also much fainter $(V=7.1$, $d=34$ '2).

H 1346 lies right between HIP 20885 ( $\theta^{1}$ Tau) and HIP 20894 $\left(\theta^{2} \mathrm{Tau}\right)$, which stars are separated by 5.6 , and is probably the combined light of the two.

H 1397, near 0.4,3.5 in Fig. C.52, lies north of HIP 9001 and HIP 9021. We identify with the nearer HIP 9001, Rybka with the slightly brighter HIP $9021(V=5.7)$, but since the separation between these two stars is just 3.3, H 1397 may correspond to the combined light of them. 
F. Verbunt and R. H. van Gent: The star catalogue of Hevelius

H 1442, near -23.1,14.5 in Fig. C.53, lies closest to HIP 58858 $(V=5.9, d=58.4)$, but we agree with Rybka that HIP 59501 (near -21.6,15.2) is the more plausibe identification, with a similar offset as H 1450 (near -20.5,12.9) to its identification HIP 60202.

H 1451, near 17.8,-7.6 in Fig. C.53, is identified by Rybka with 40 Vir $=\psi$ Vir, which however is the identification of $\mathrm{H} 1437$.

$\mathrm{H} 1460$, near 25.3,4.8 in Fig. C.54, is the combined light of HIP 63121 and HIP 63125, separated by $19^{\prime \prime}$.

H 1499, near -21.5,6.7 in Fig. C.54, is unidentified.

$\mathrm{H} 1505$ and $\mathrm{H} 1519$, respectively near $10.3,-9.5$ and $9.3,-8.5$ in Fig. C.54, are identified by Rybka with HIP 54136 and HIP 53838, respectively near 10.3,-10.2 and 9.2,-9.8. This is an attractive solution, but the offsets are large and we consider it uncertain.

H 1521, near 0.2,11.3 in Fig. C.54 lies close to both HIP 56035, which we suggest as the identification, and HIP 56290, slighly brighter but further ( $V=5.5, d=43$ '. $)$, suggested by Rybka as the identification.

H 1536, near 10.4,3.4 in Fig. C.55, is identified by Rybka with HIP 74605, near 8.6,4.6, which is a close match for H 1537, and identified by us accordingly.

H 1537, near 8.7,4.6 in Fig. C.55, is identified by Rybka with HIP $74272(V=6.2, d=38$ '. $)$.

H 1540, near -5.4,5.7 in Fig. C.56, in Nova Vul 1670, whose modern counterpart CK Vul was discovered at $m_{R}=20.7$ in 1983 (Shara et al. 1985). We use the position given in that paper to compute the offset with respect to the position given by Hevelius, since CK Vul is too faint for the Hipparcos catalogue. $\mathrm{H} 1561$, near $-12.7,0.9$ in Fig. C.56, is close to three stars. We identify it with the nearest. The brightest of the three, HIP 95498 ( $V=5.1, d=16^{\prime} 6$ ) is too far, the identification suggested by Rybka, HIP95582 ( $\left.V=5.8, d=15{ }^{\prime} 9\right)$, is both too far and too faint.

\section{Appendix C: Figures}

To illustrate and clarify our identifications we provide a Figure for each constellation. In these figures the stars listed with the constellation in Hevelius are shown red when Hevelius gives them an $O T$ value $(>0)$ indicating their presence in Kepler or in Secunda classis, and blue otherwise $(O T=0)$.

Other stars listed in Hevelius are light-red $(O T>0)$ and light-blue $(O T=0)$, respectively. To minimize deformation of the constellations, we determine the center of the constellation $\lambda_{\mathrm{c}}, \beta_{\mathrm{c}}$ from the extremes in $\lambda$ and $\beta$, compute the rotation matrix which moves this center to $(x, y, z)=(1,0,0)$, and then apply this rotation to each of the stellar positions $\lambda_{i}, \beta_{i}$. (For exact details see Paper I.) The resulting $y, z$ values correspond roughly to differences in longitude and latitude, exact at the center $\lambda_{\mathrm{c}}, \beta_{\mathrm{c}}$ and increasingly deformed away from the center. We plot the rotated positions of the stars in Hevelius as $d \lambda \equiv y$ and $d \beta \equiv z$ with filled circles. The same rotation matrix is applied to all stars down to a magnitude limit $V_{\mathrm{m}}$ (usually $V_{m}=6.0$ ) from the Hipparcos Catalogue and those in the field of view are plotted as open circles. The symbol sizes are determined from the magnitudes as indicated in the legenda. The values used for $\lambda_{\mathrm{c}}, \beta_{\mathrm{c}}$ and $V_{\mathrm{m}}$ are indicated with each figure.

Where necessary we show enlarged detail Figures; for easy comparison with the figures showing the whole constellation, these detail figures use the same rotation center (and thus rotation matrix).

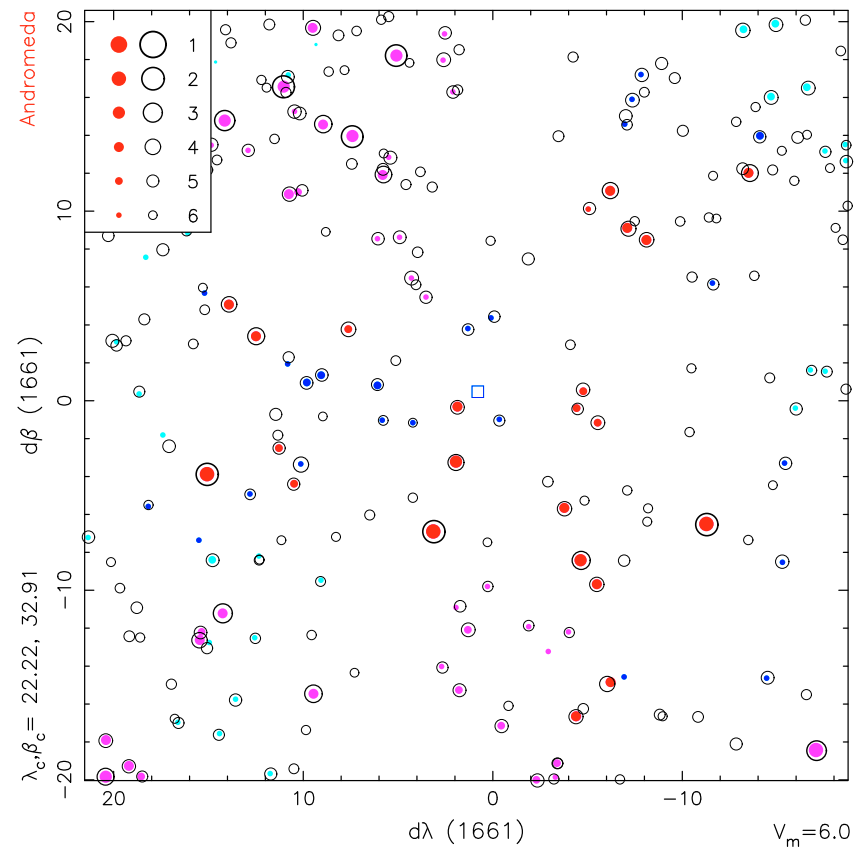

Fig. C.1. Andromeda. The nebulous object near the center is M31.

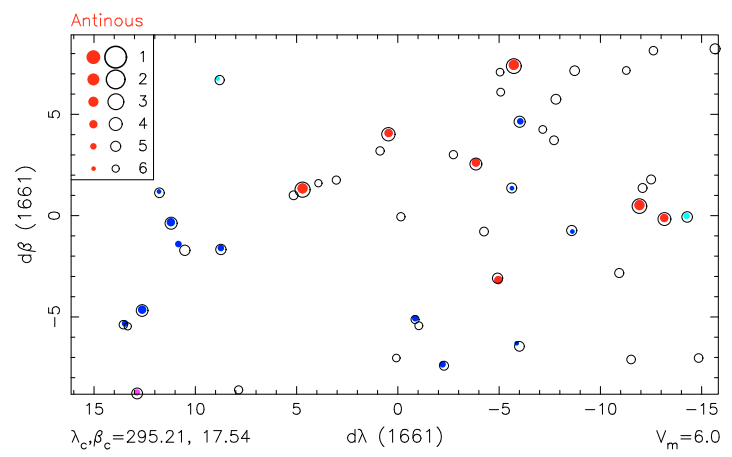

Fig. C.2. Antinous.

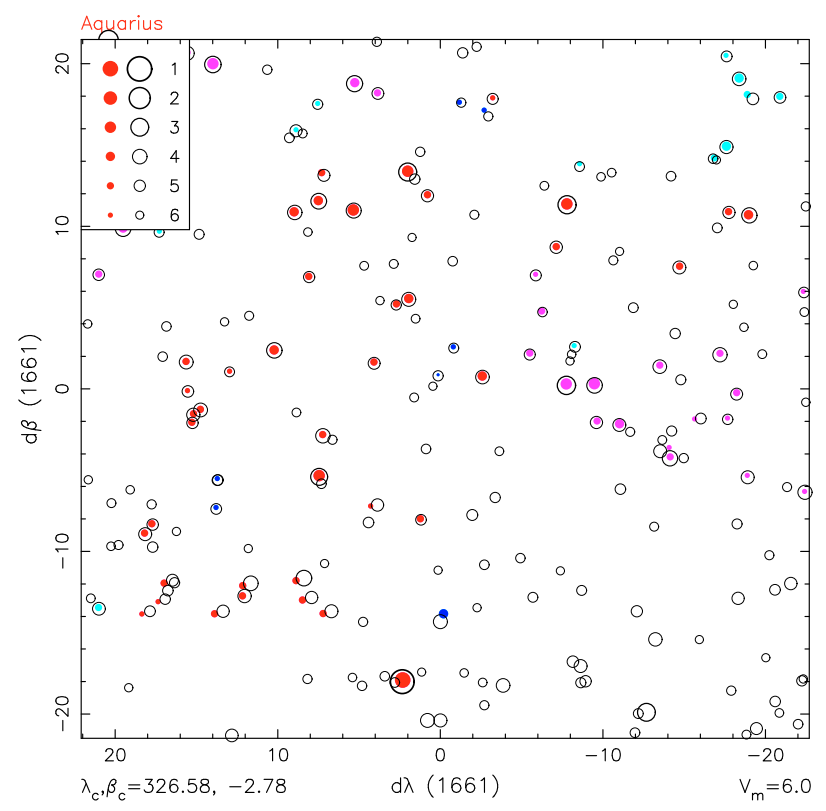

Fig. C.3. Aquarius. 
A\&A 516, A29 (2010)
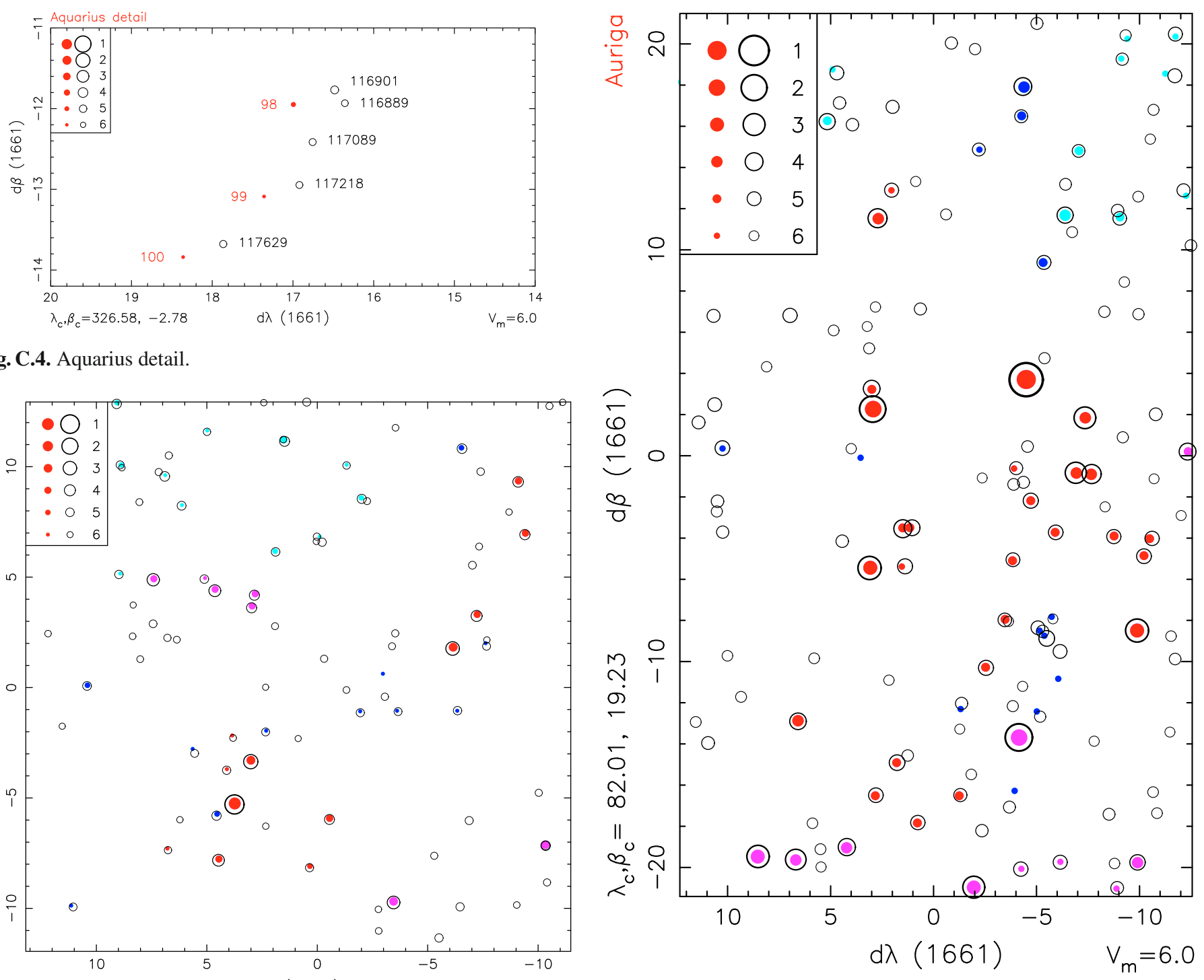

Fig. C.4. Aquarius detail.

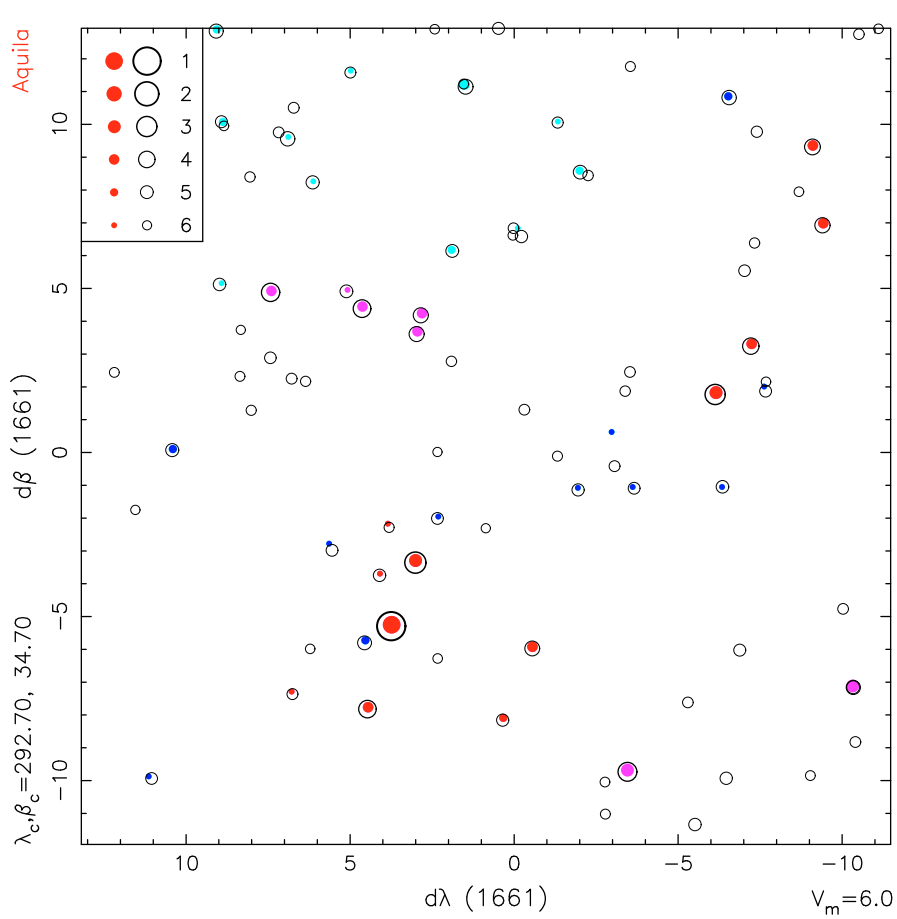

Fig. C.7. Auriga.

Fig. C.5. Aquila.
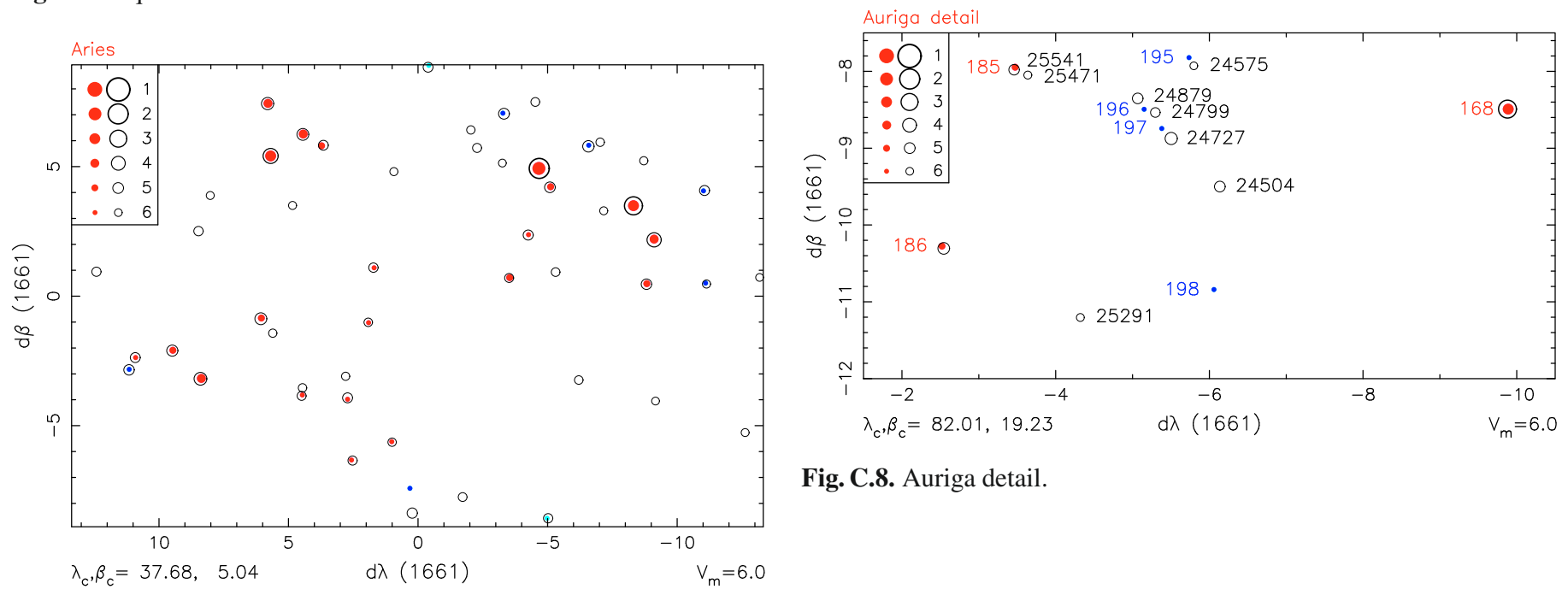

Fig. C.8. Auriga detail.

Fig. C.6. Aries. 
F. Verbunt and R. H. van Gent: The star catalogue of Hevelius

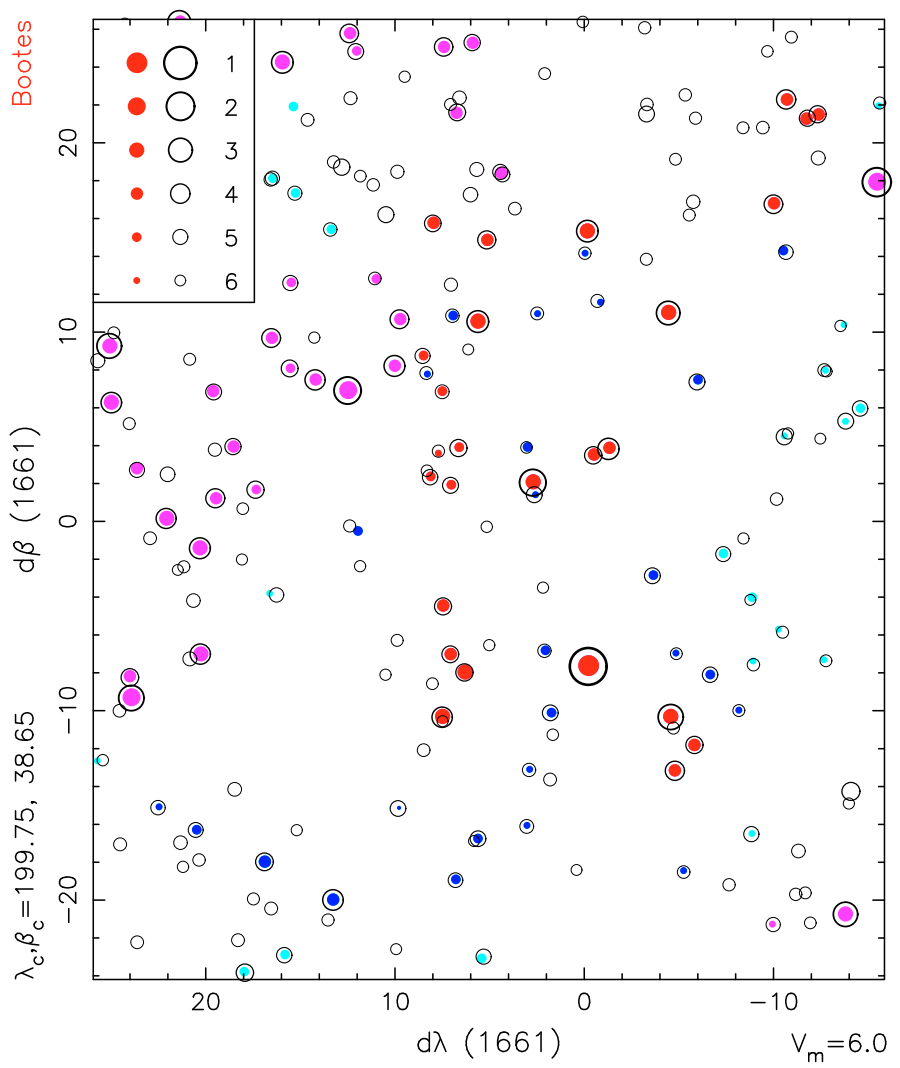

Fig. C.9. Bootes.

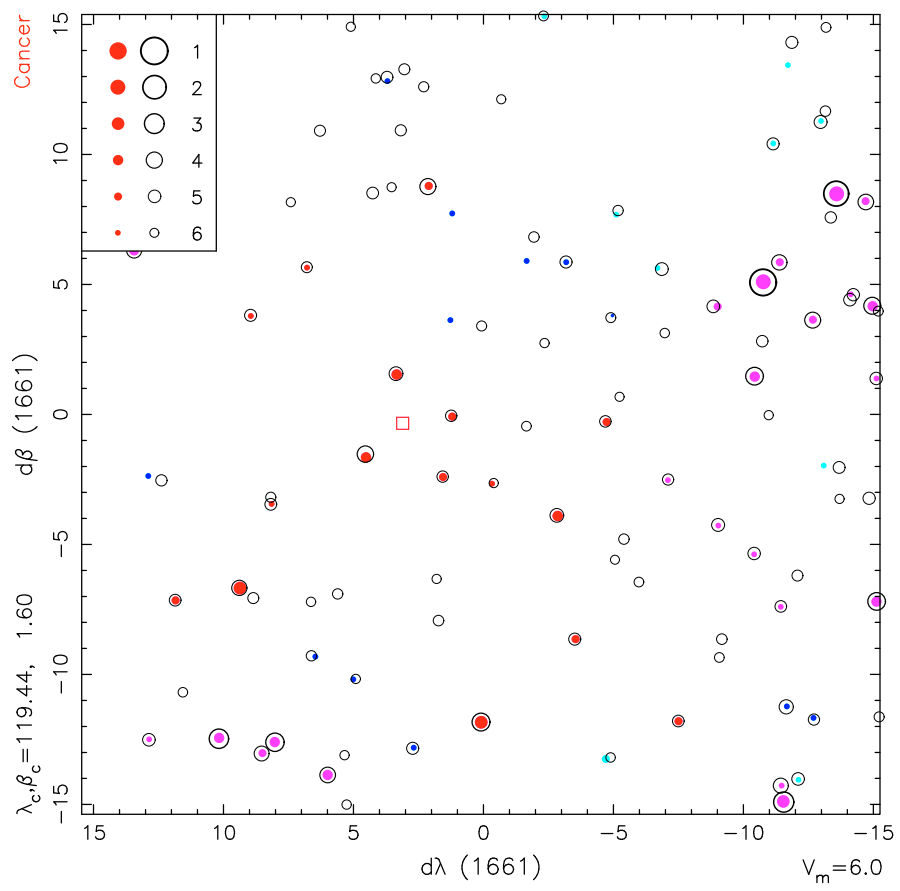

Fig. C.10. Cancer.

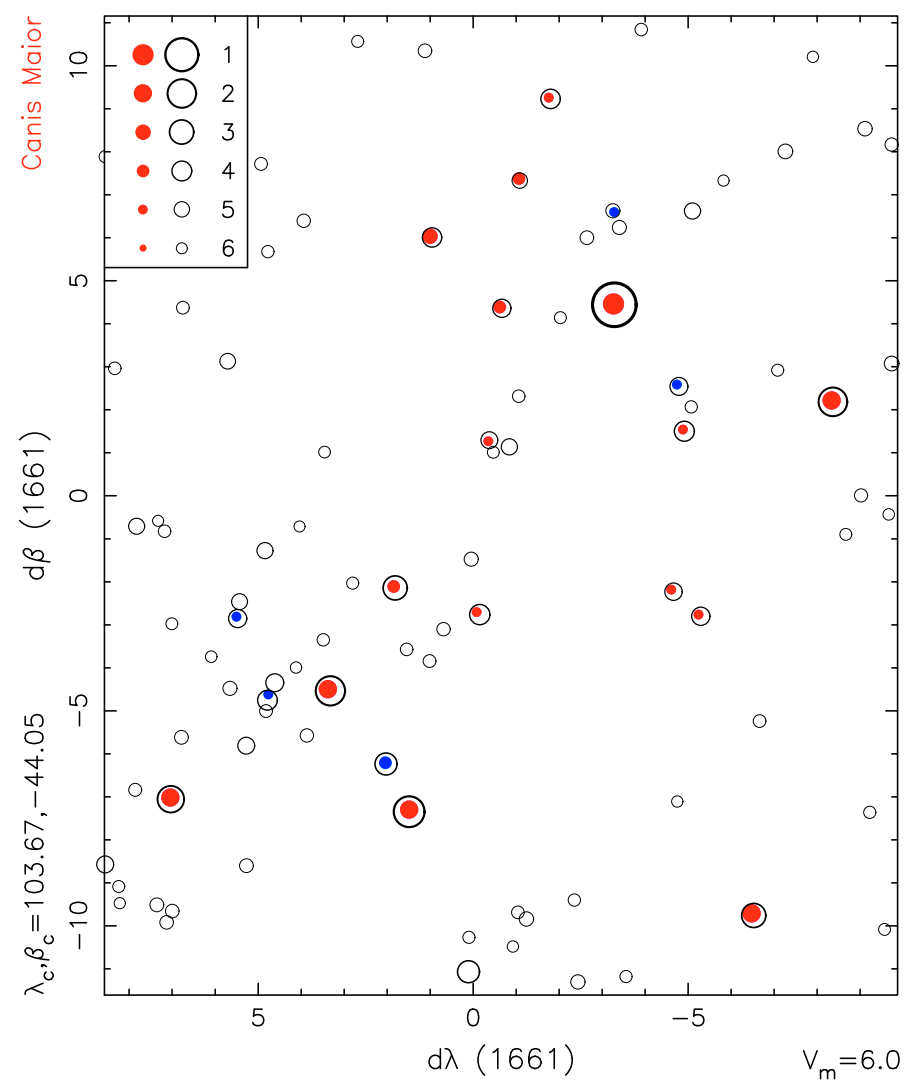

Fig. C.11. Canis Maior.

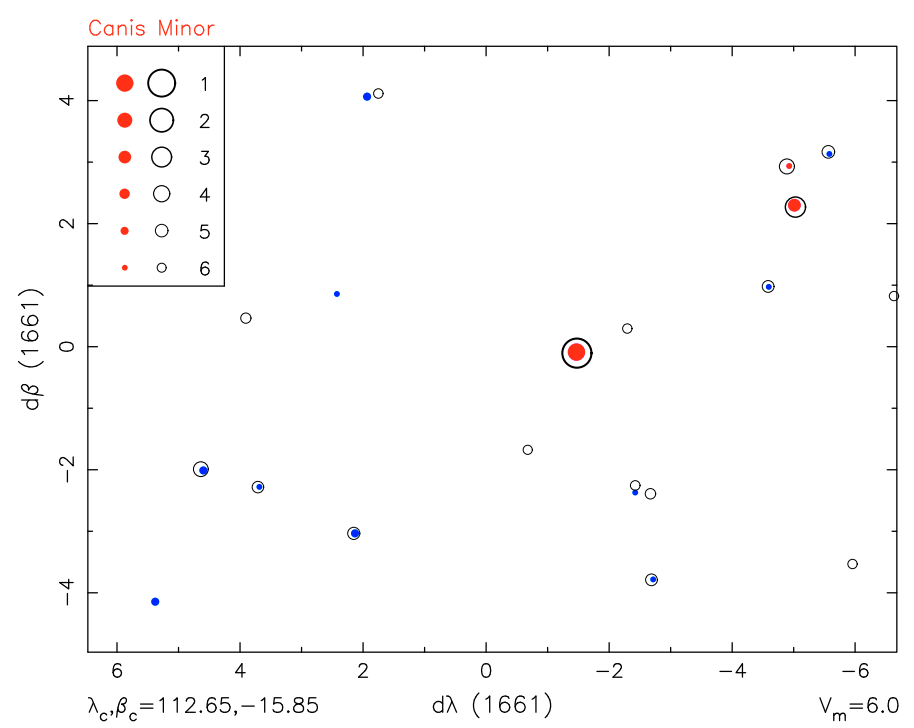

Fig. C.12. Canis Minor. 
A\&A 516, A29 (2010)
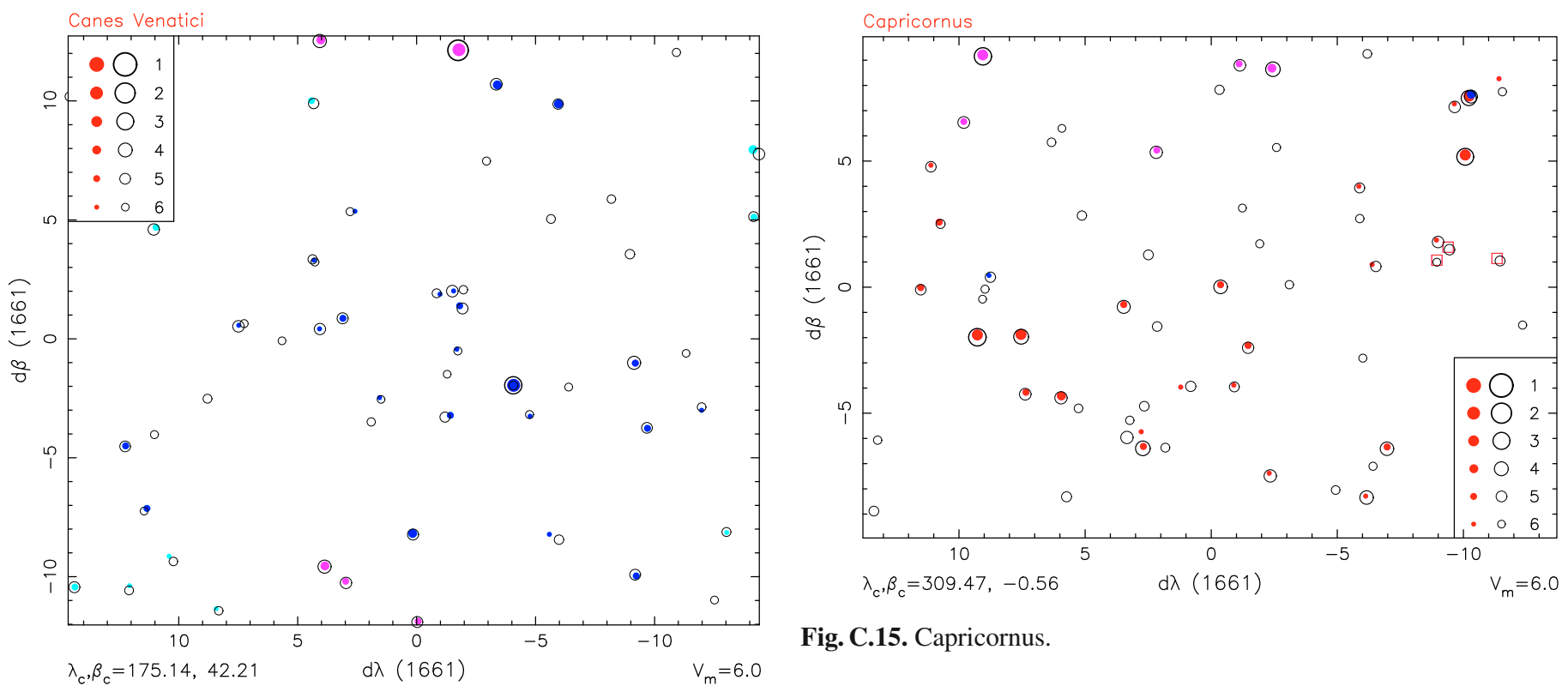

Fig. C.15. Capricornus.

Fig. C.13. Canes Venatici.
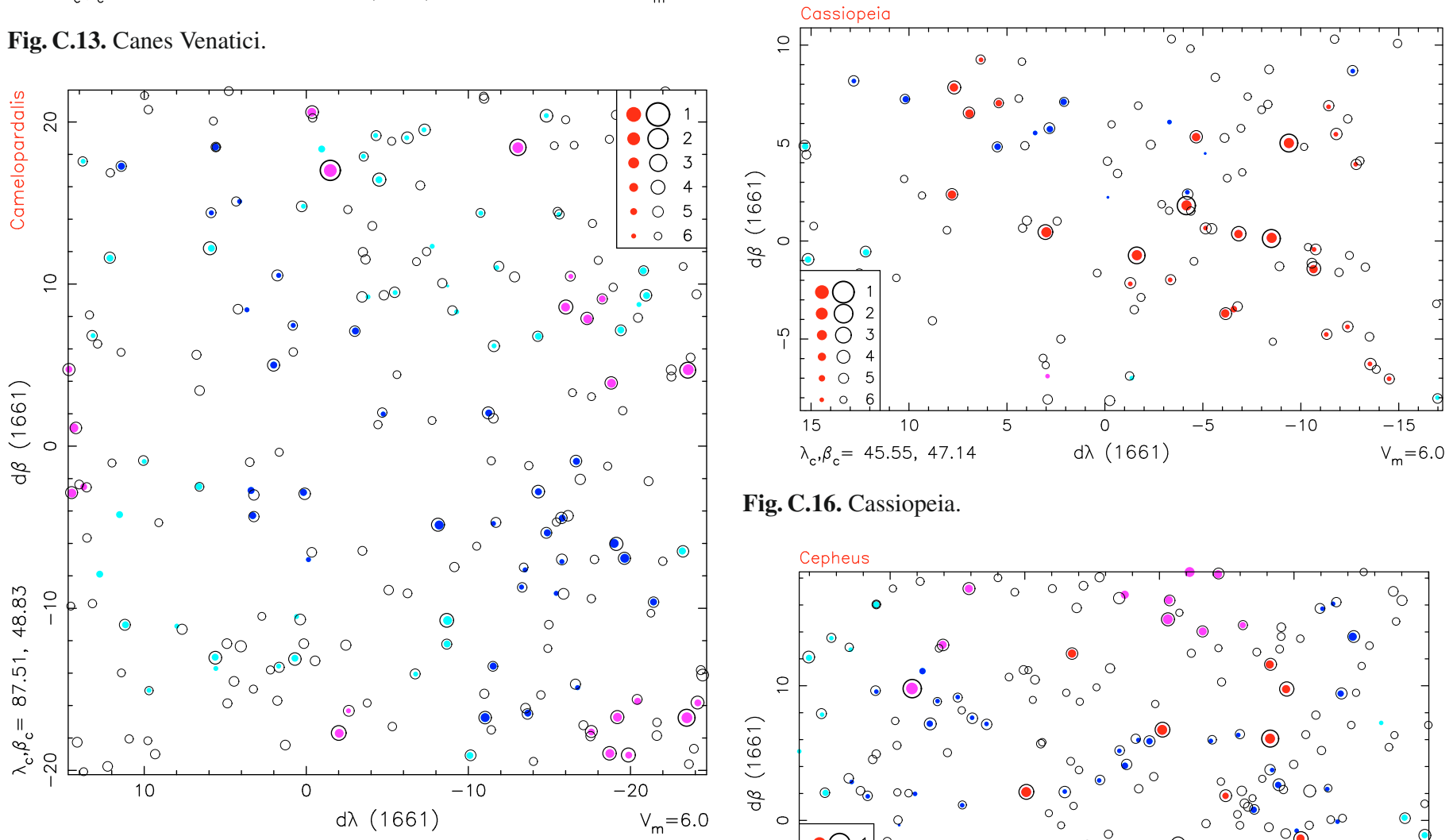

Fig. C.16. Cassiopeia.

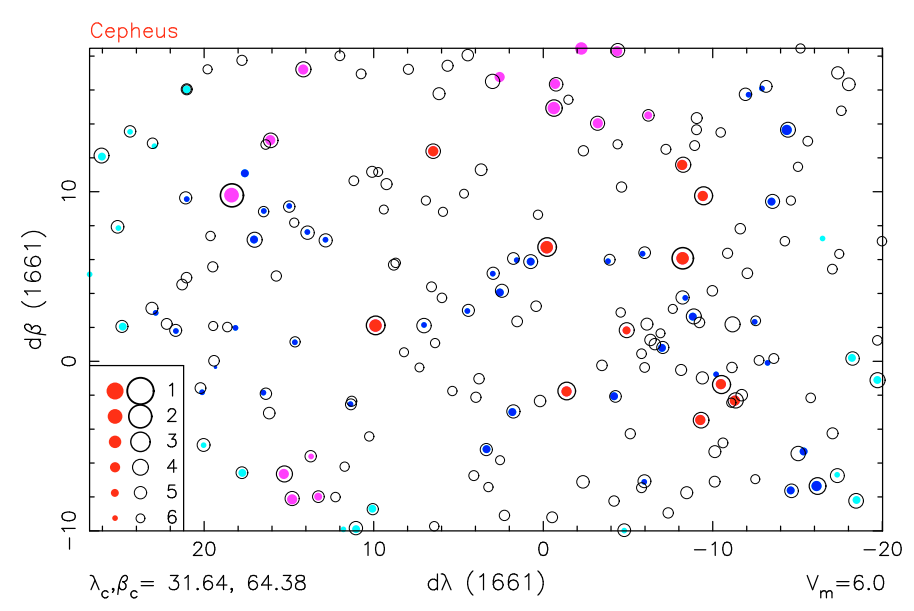

Fig. C.17. Cepheus. 
F. Verbunt and R. H. van Gent: The star catalogue of Hevelius
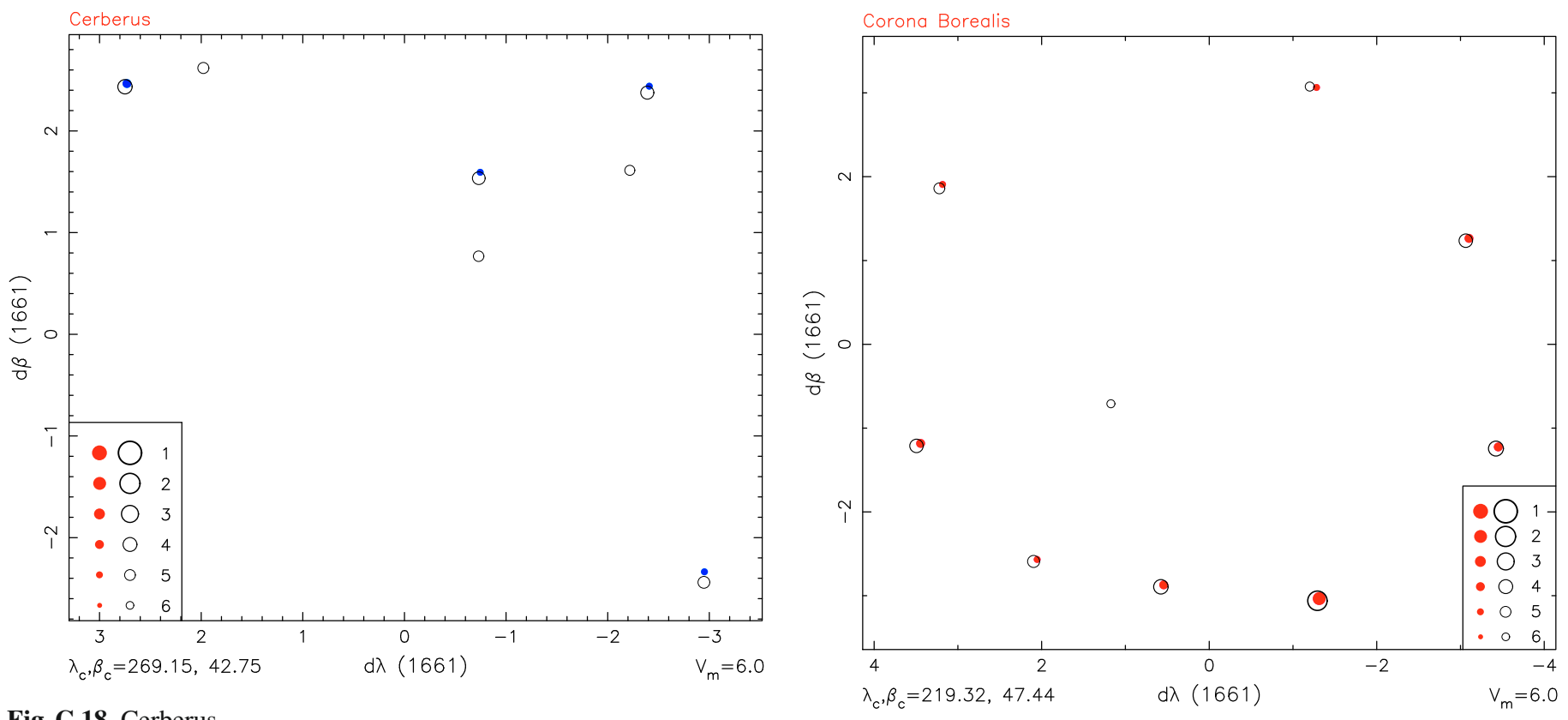

Fig. C.18. Cerberus.

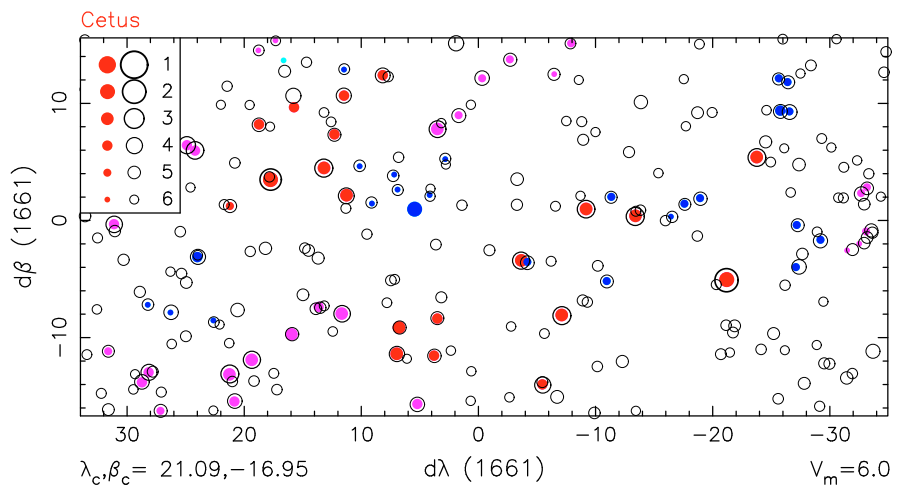

Fig. C.21. Corona Borealis.

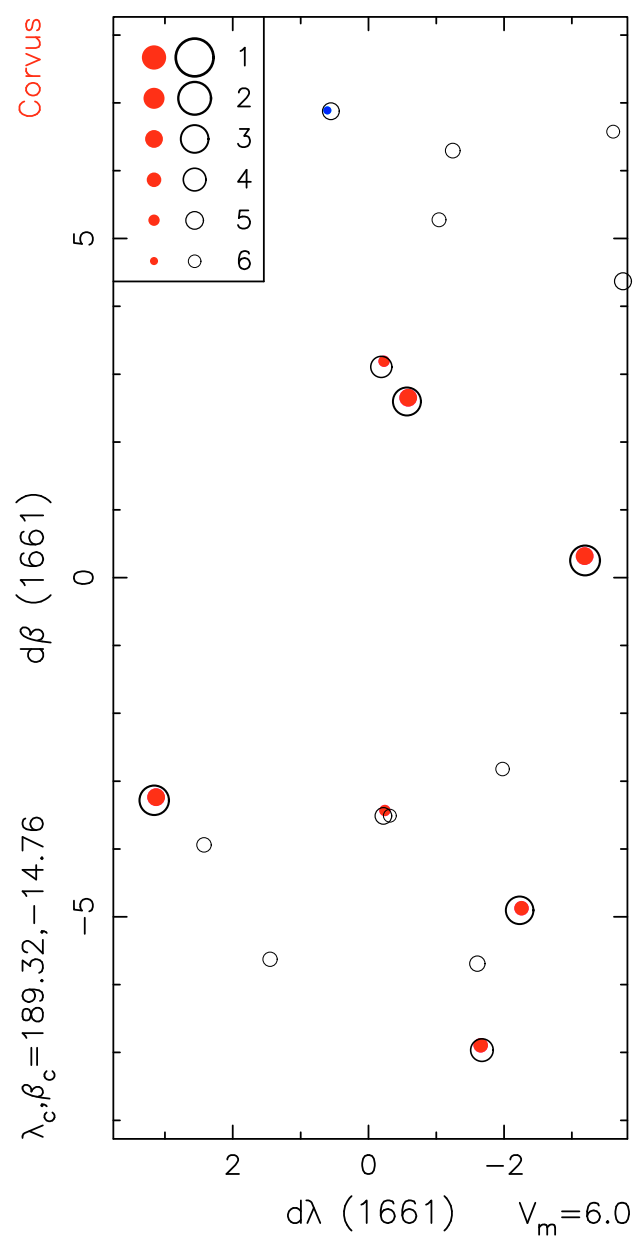

Fig. C.20. Coma Berenices.

Fig. C.22. Corvus. 
A\&A 516, A29 (2010)
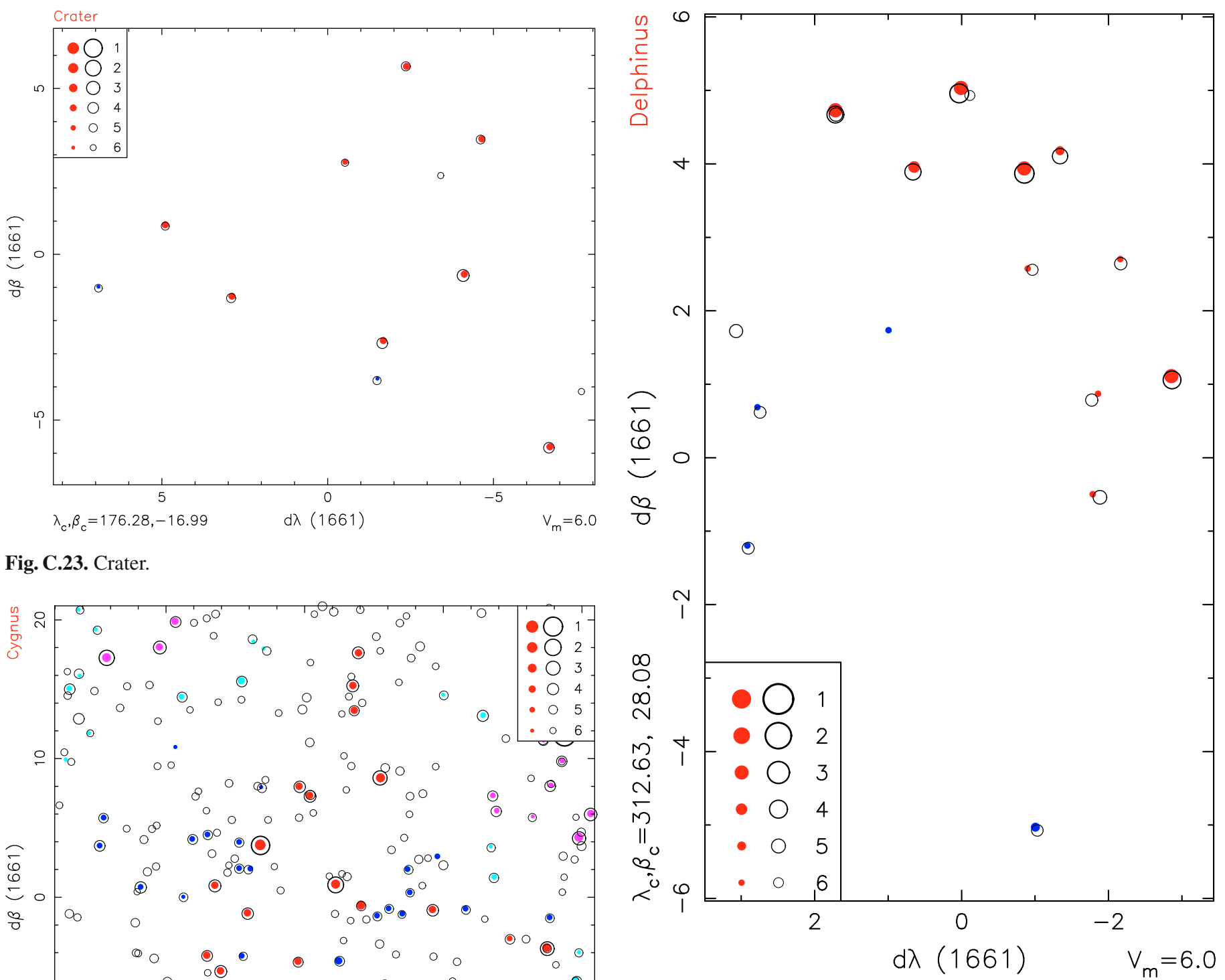

Fig. C.23. Crater.

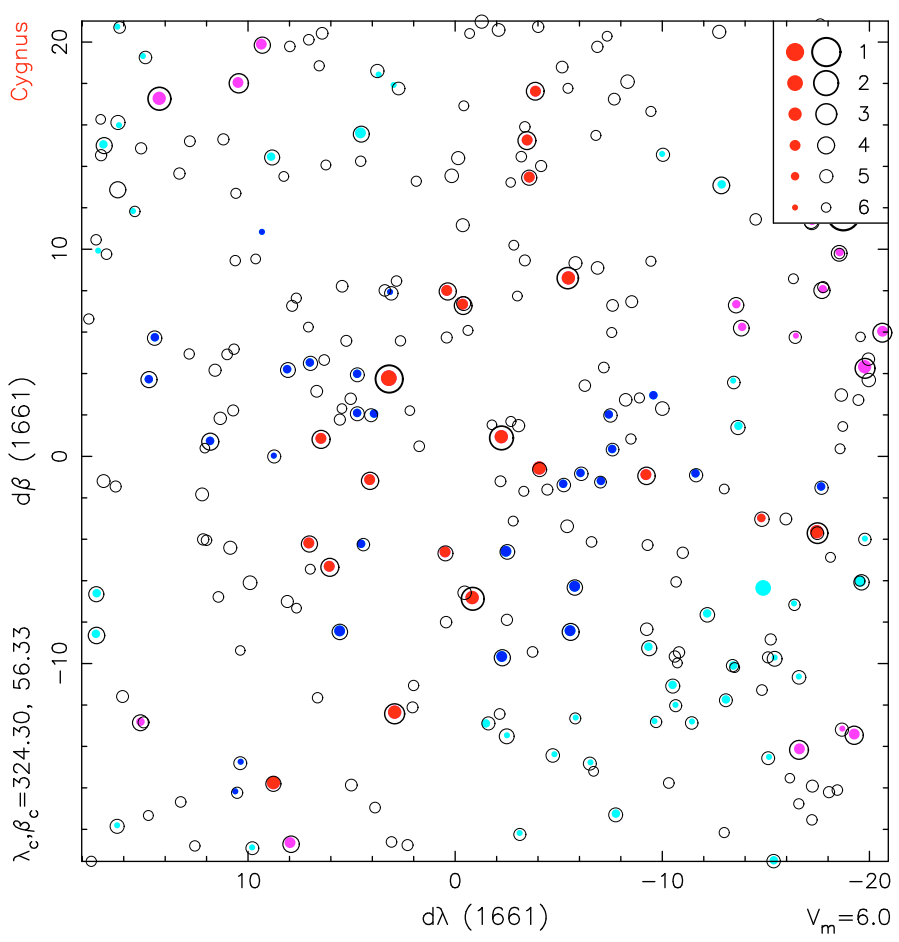

Fig. C.24. Cygnus.

Fig. C.25. Delphinus.

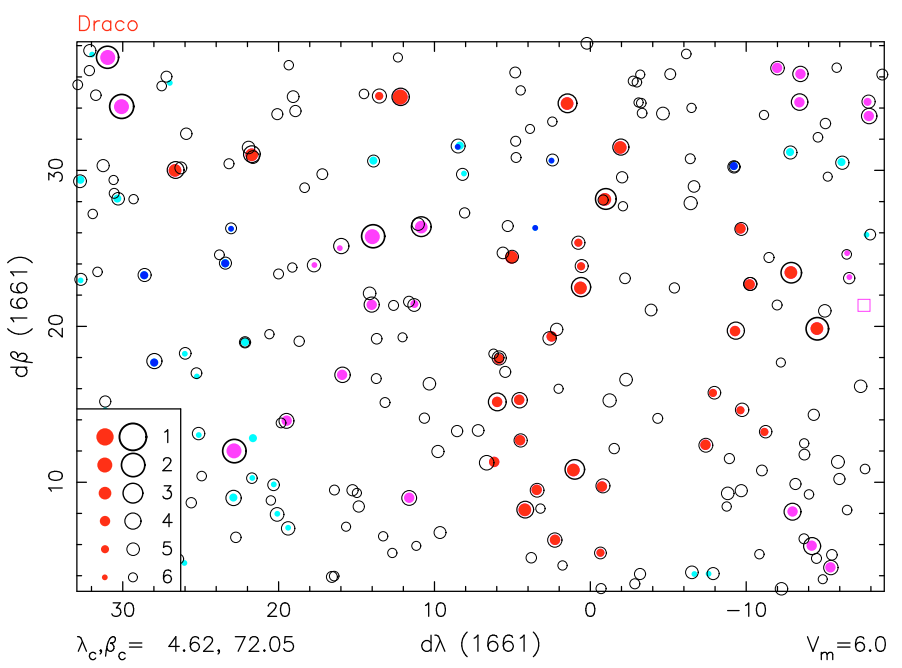

Fig. C.26. Draco. 
F. Verbunt and R. H. van Gent: The star catalogue of Hevelius

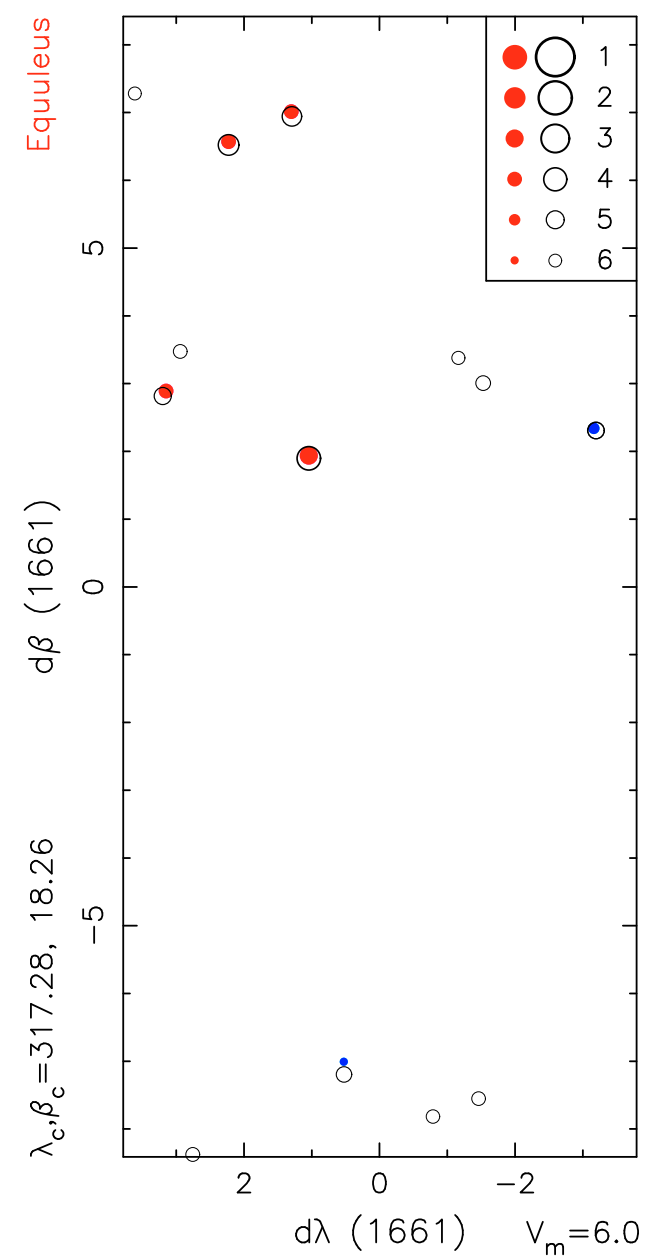

Fig. C.27. Equuleus.

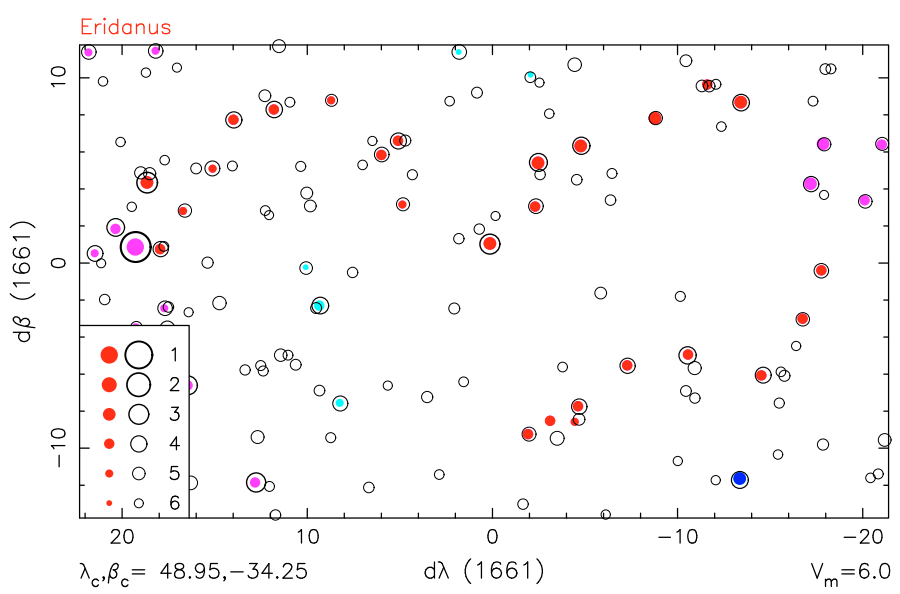

Fig. C.28. Eridanus.

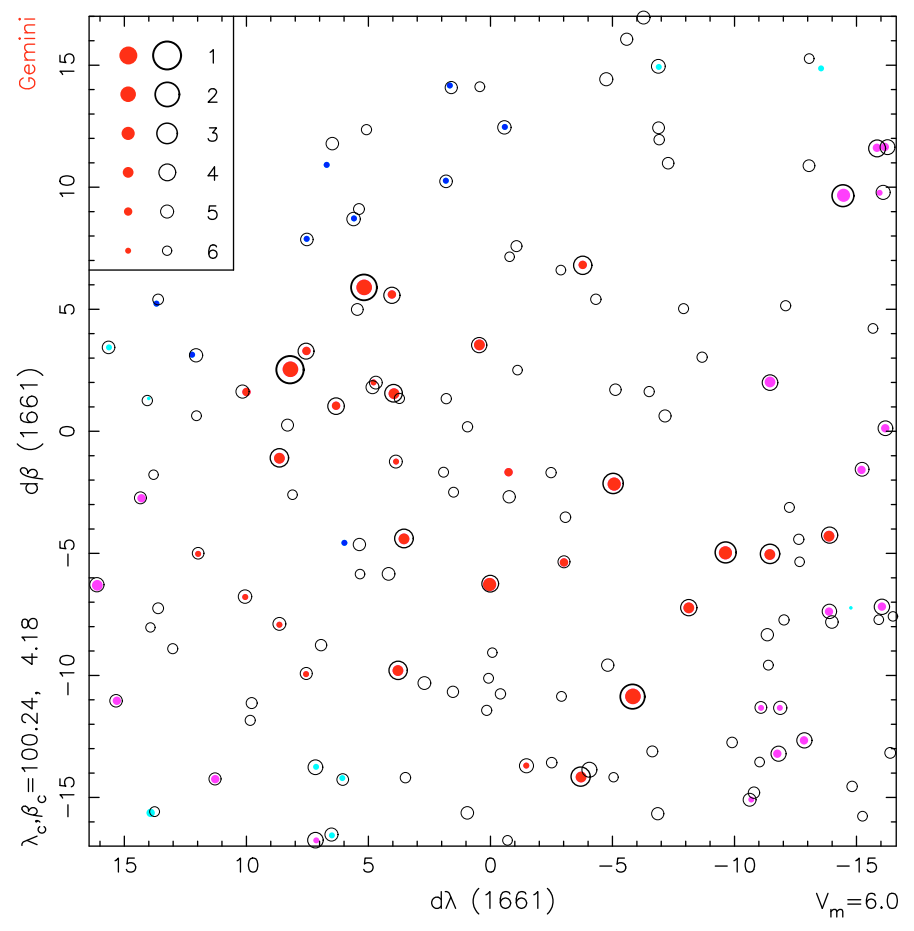

Fig. C.29. Gemini.

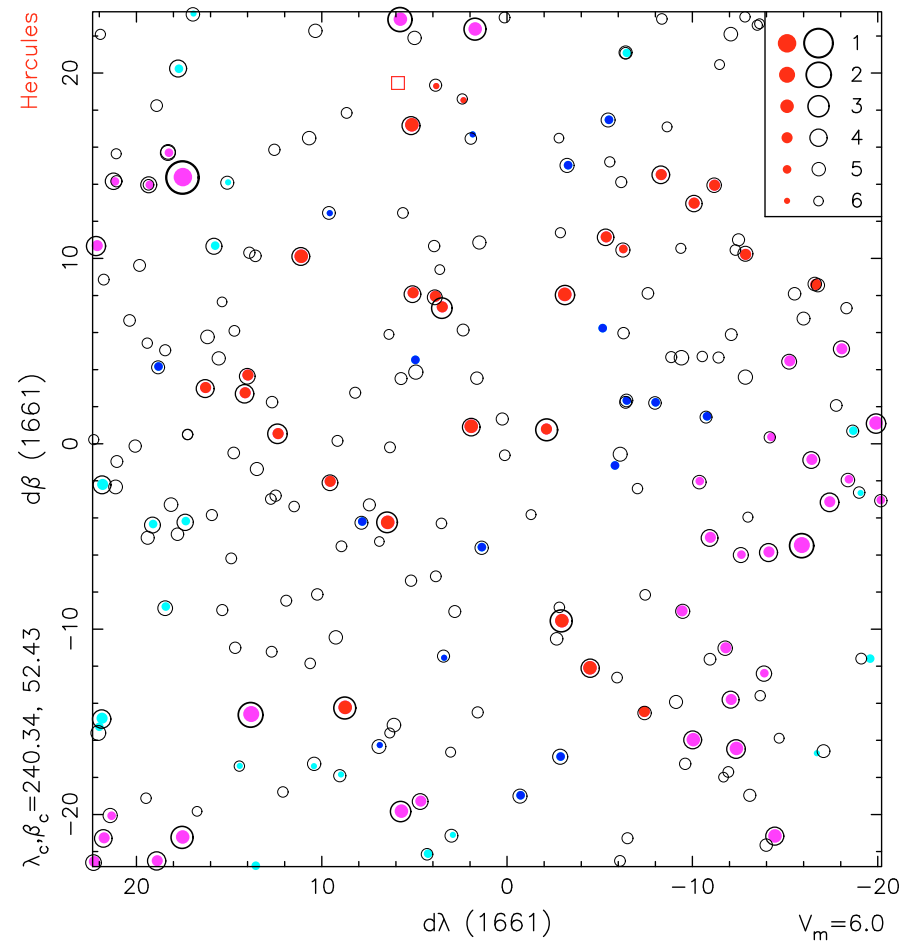

Fig. C.30. Hercules. 
A\&A 516, A29 (2010)

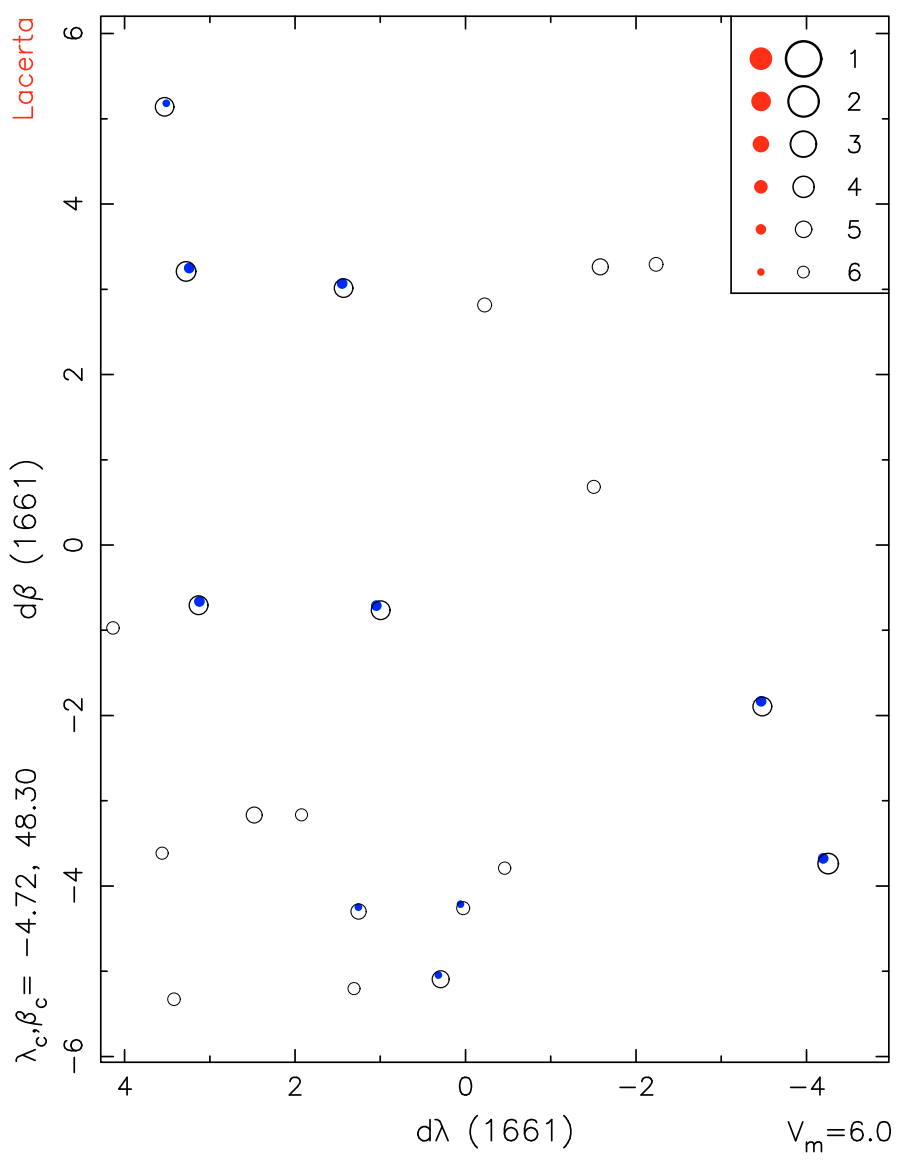

Fig. C.31. Lacerta.

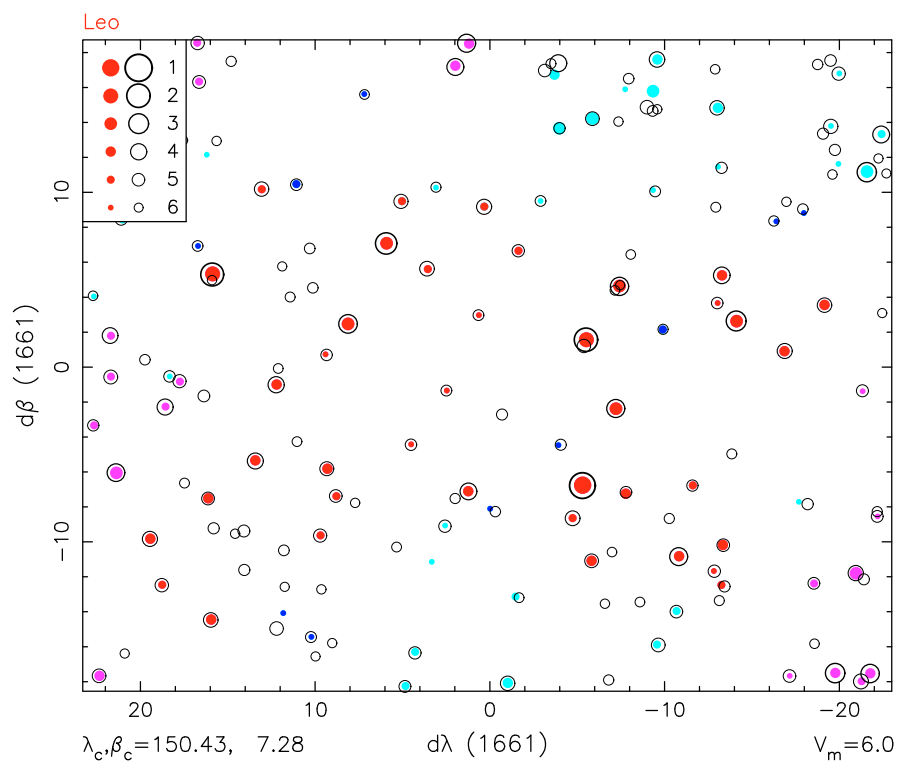

Fig. C.32. Leo.

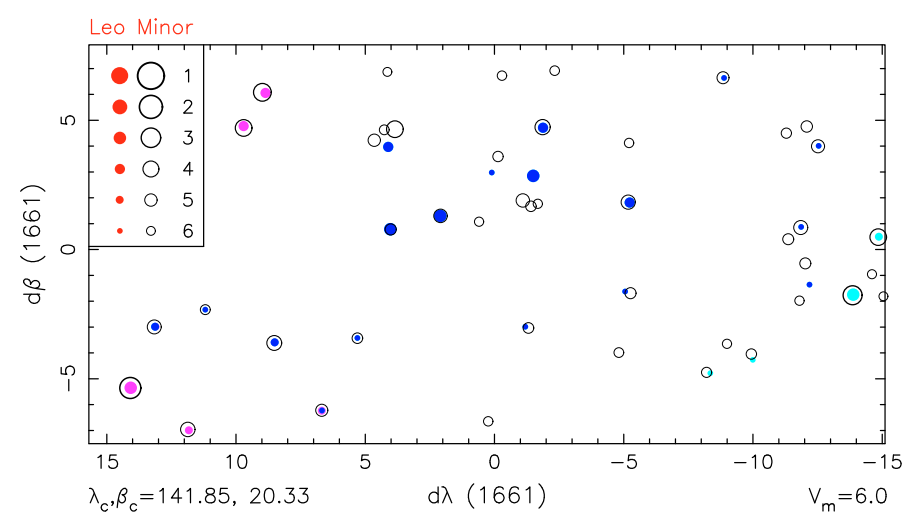

Fig. C.33. Leo Minor.

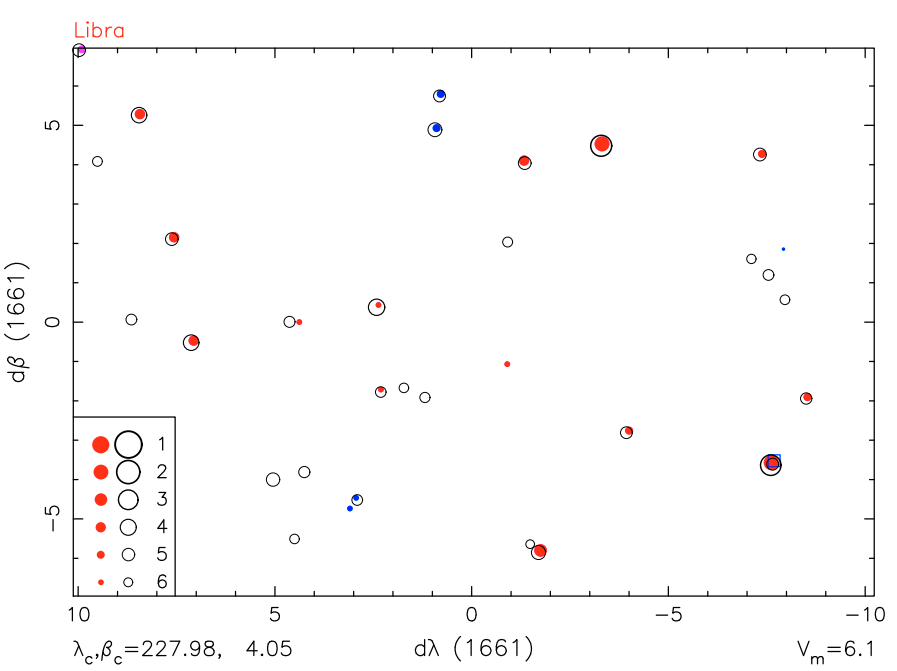

Fig. C.34. Libra.

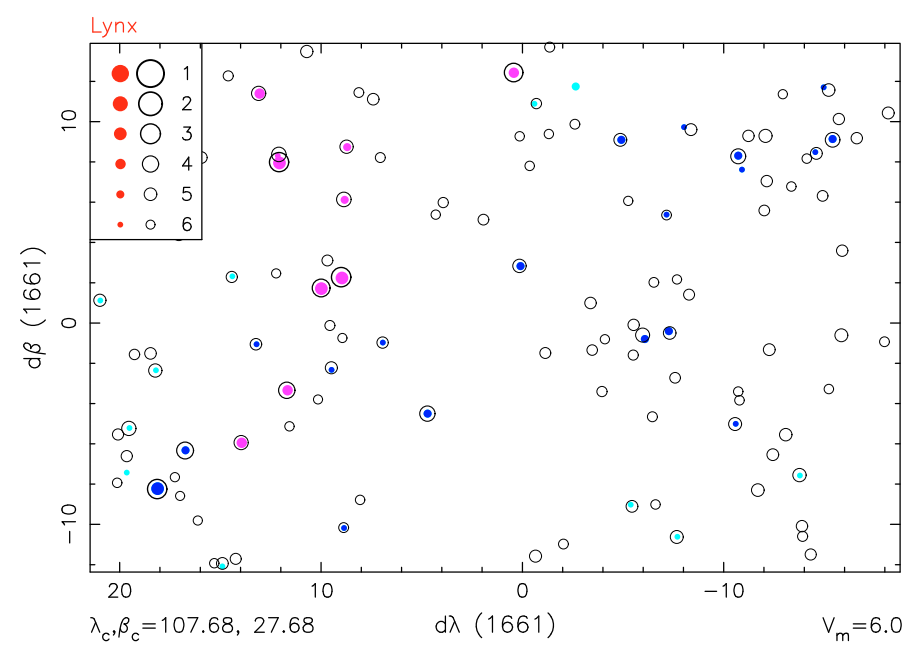

Fig. C.35. Lynx. 
F. Verbunt and R. H. van Gent: The star catalogue of Hevelius
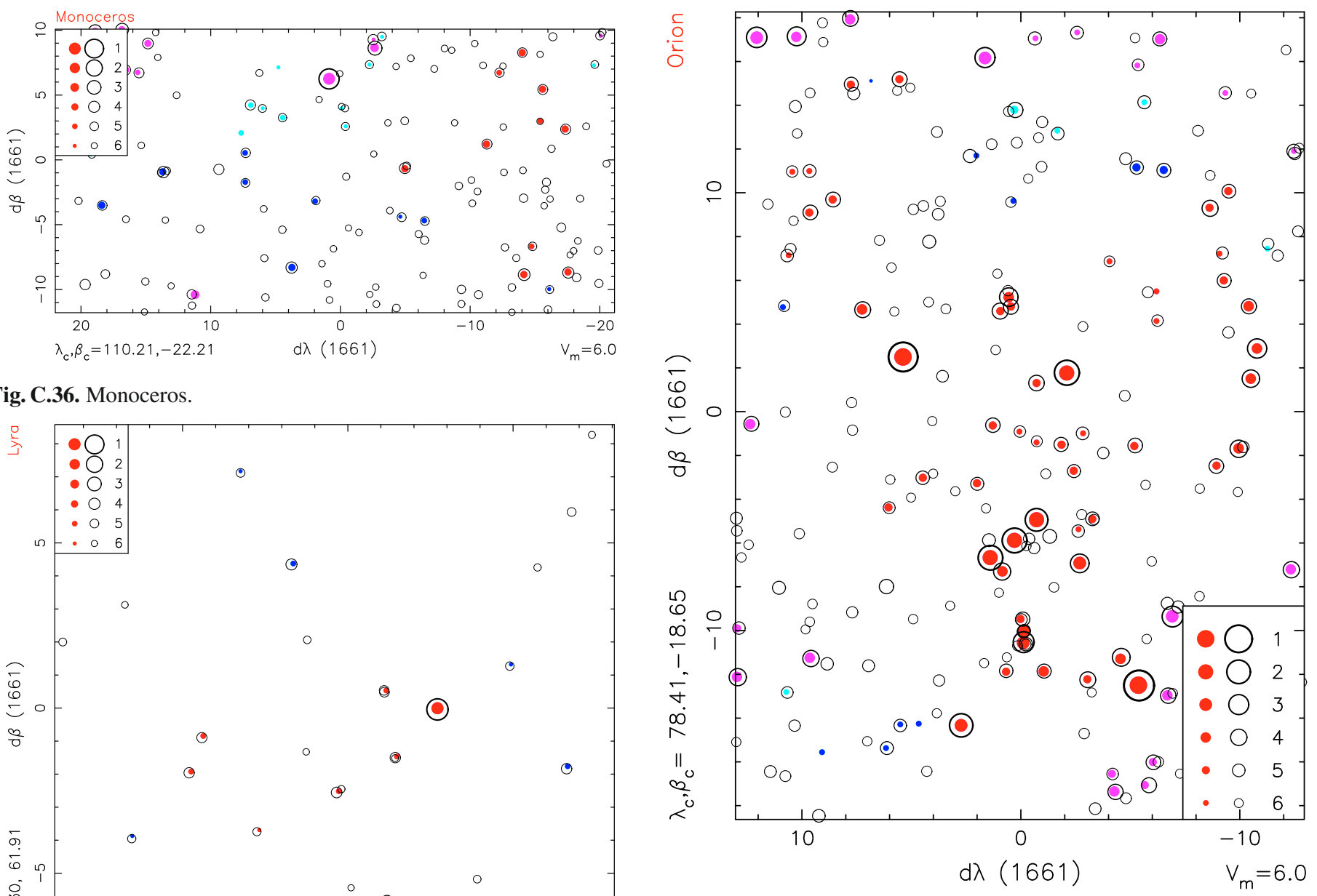

Fig. C.36. Monoceros.

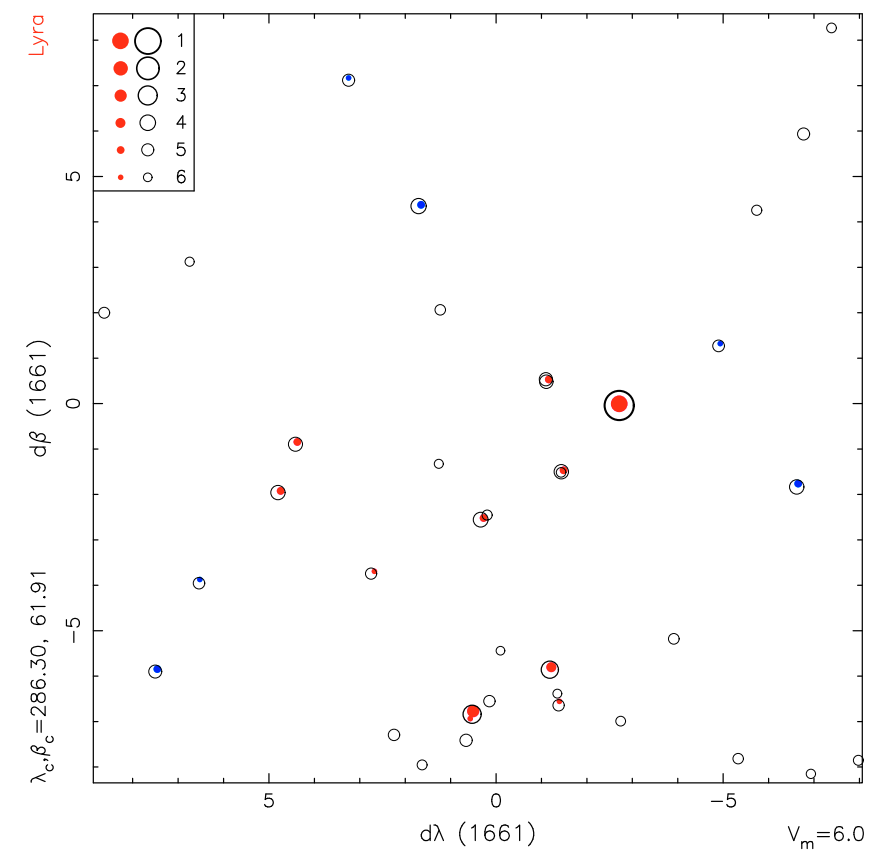

Fig. C.37. Lyra.

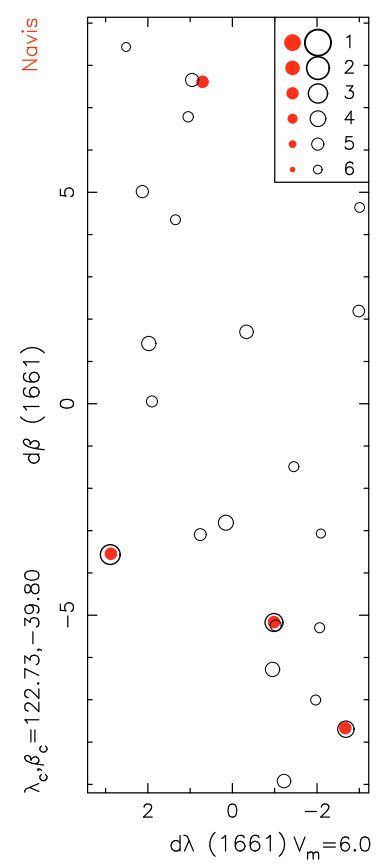

Fig. C.39. Orion.

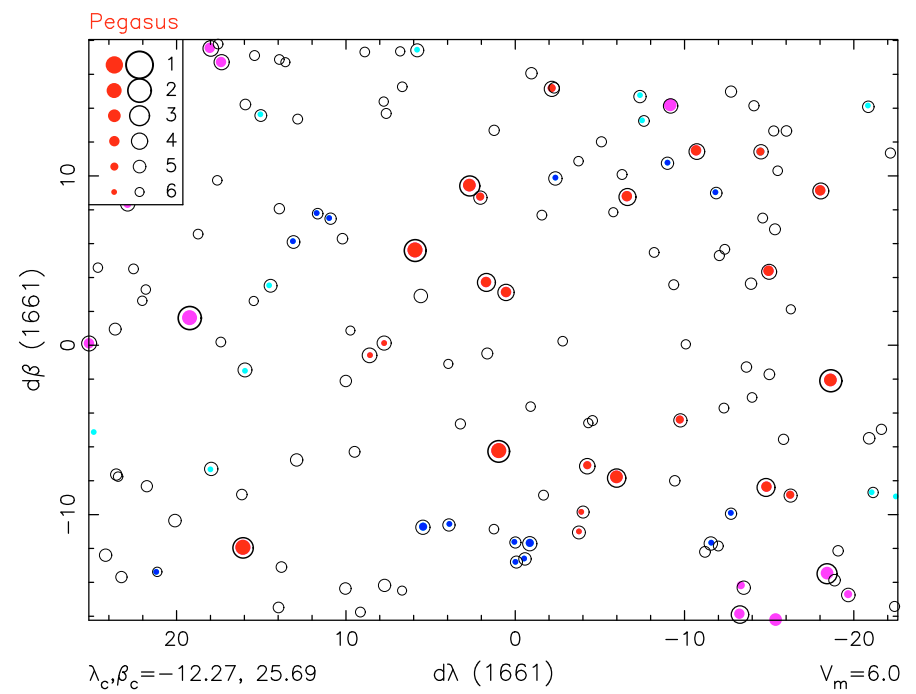

Fig. C.40. Pegasus. 
A\&A 516, A29 (2010)
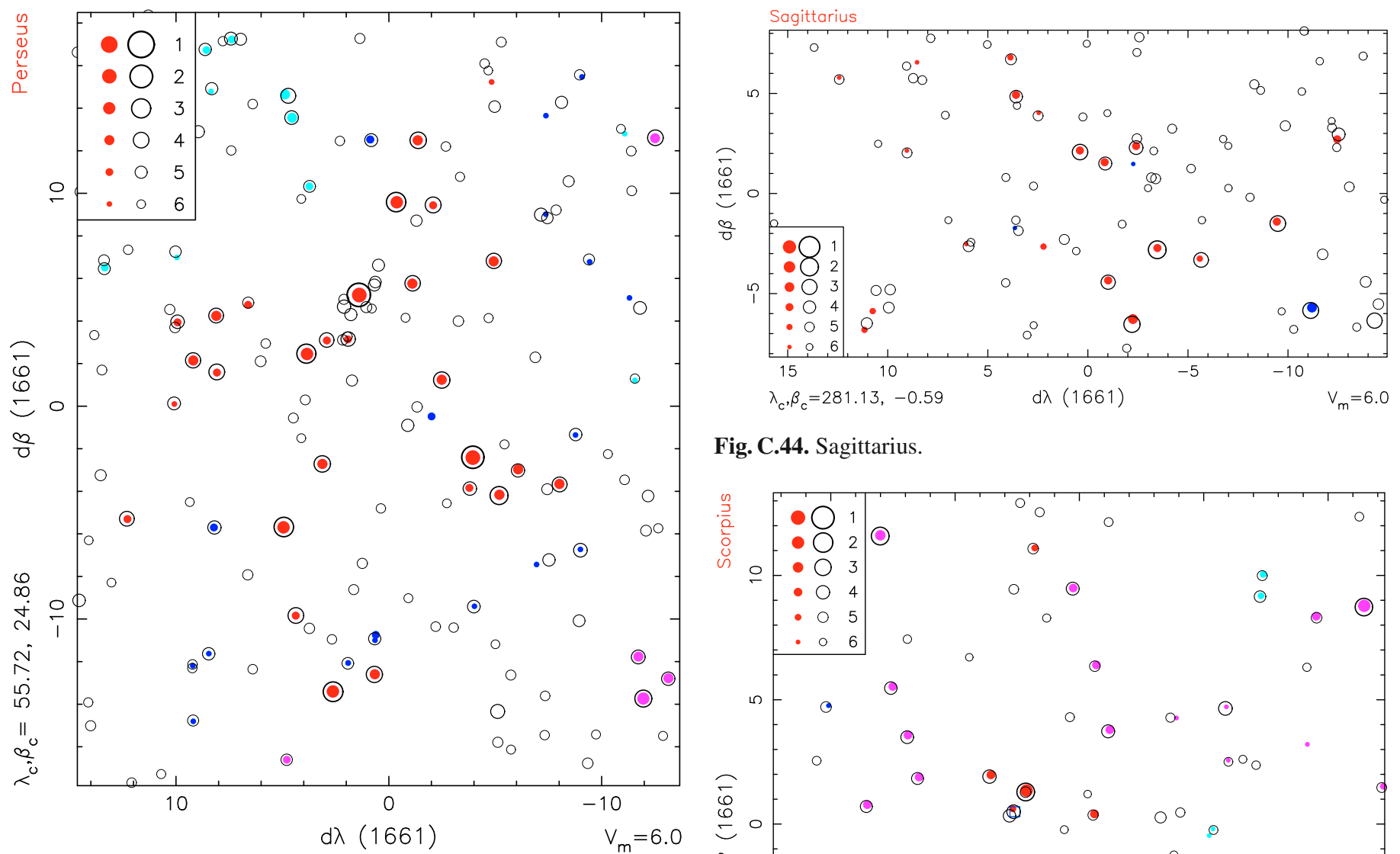

Fig. C.44. Sagittarius.

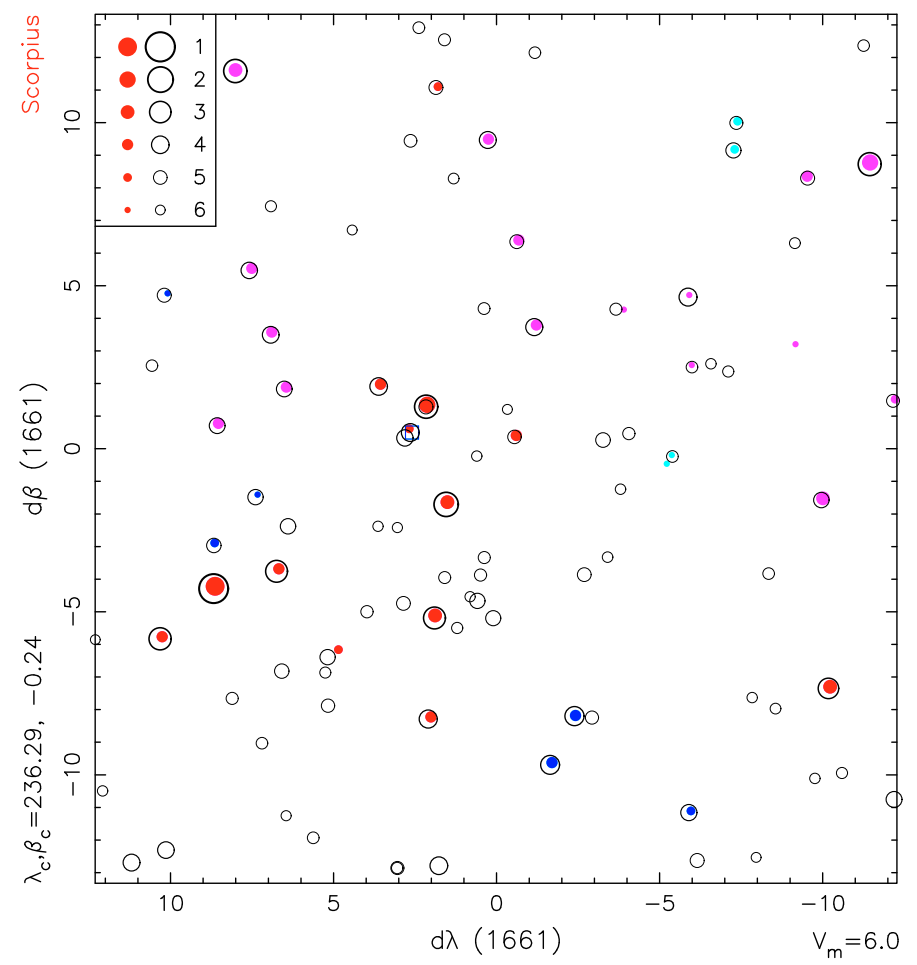

Fig. C.41. Perseus.

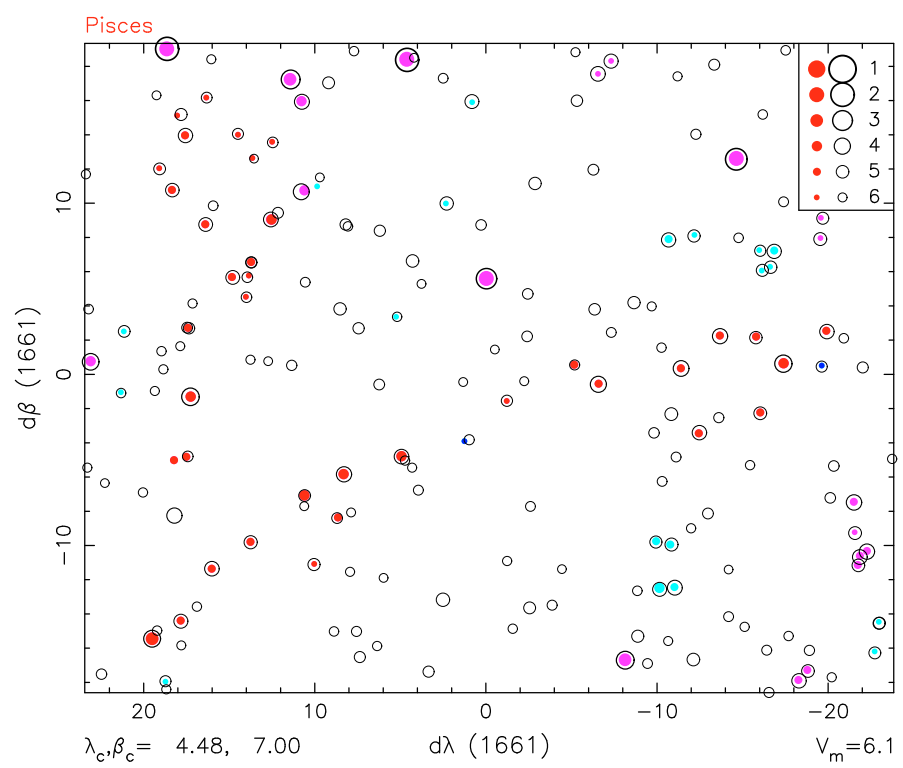

Fig. C.42. Pisces.

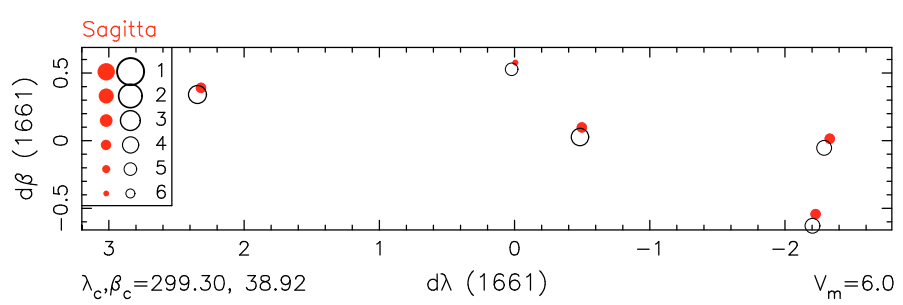

Fig. C.45. Scorpius.

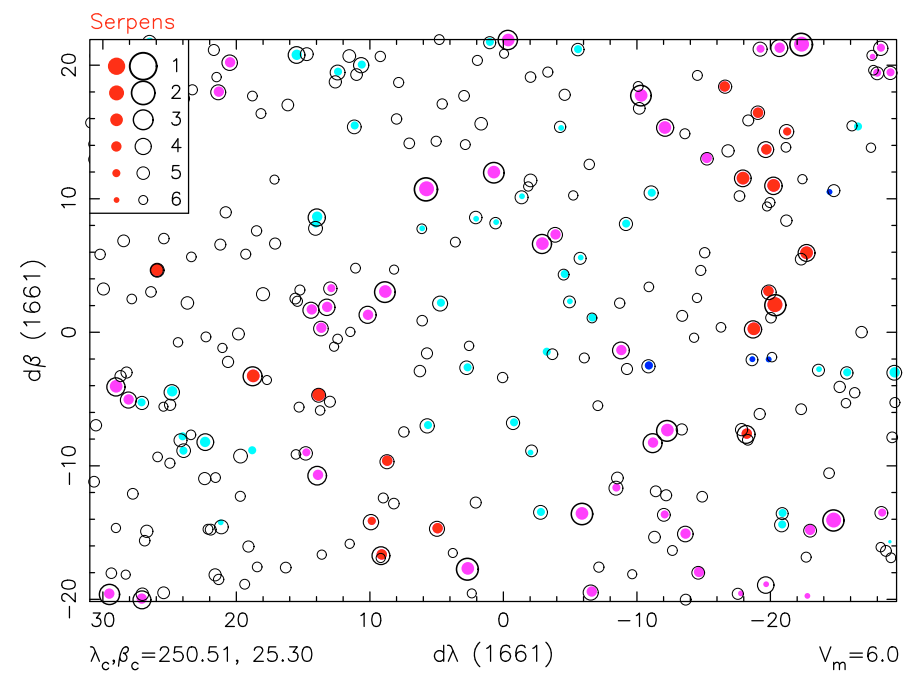

Fig. C.46. Serpens. 
F. Verbunt and R. H. van Gent: The star catalogue of Hevelius
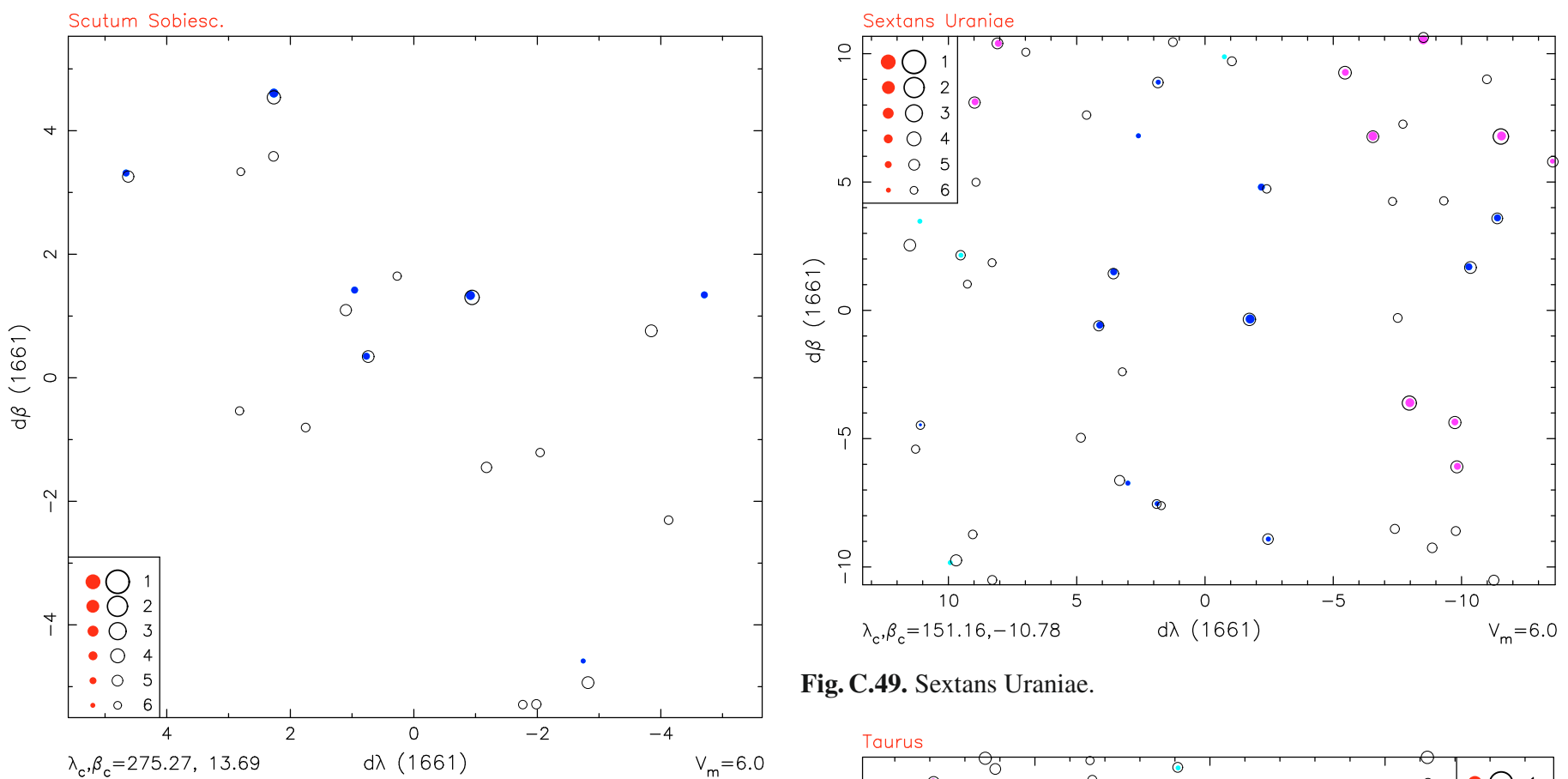

Fig. C.49. Sextans Uraniae.

Fig. C.47. Scutum Sobiescanum.
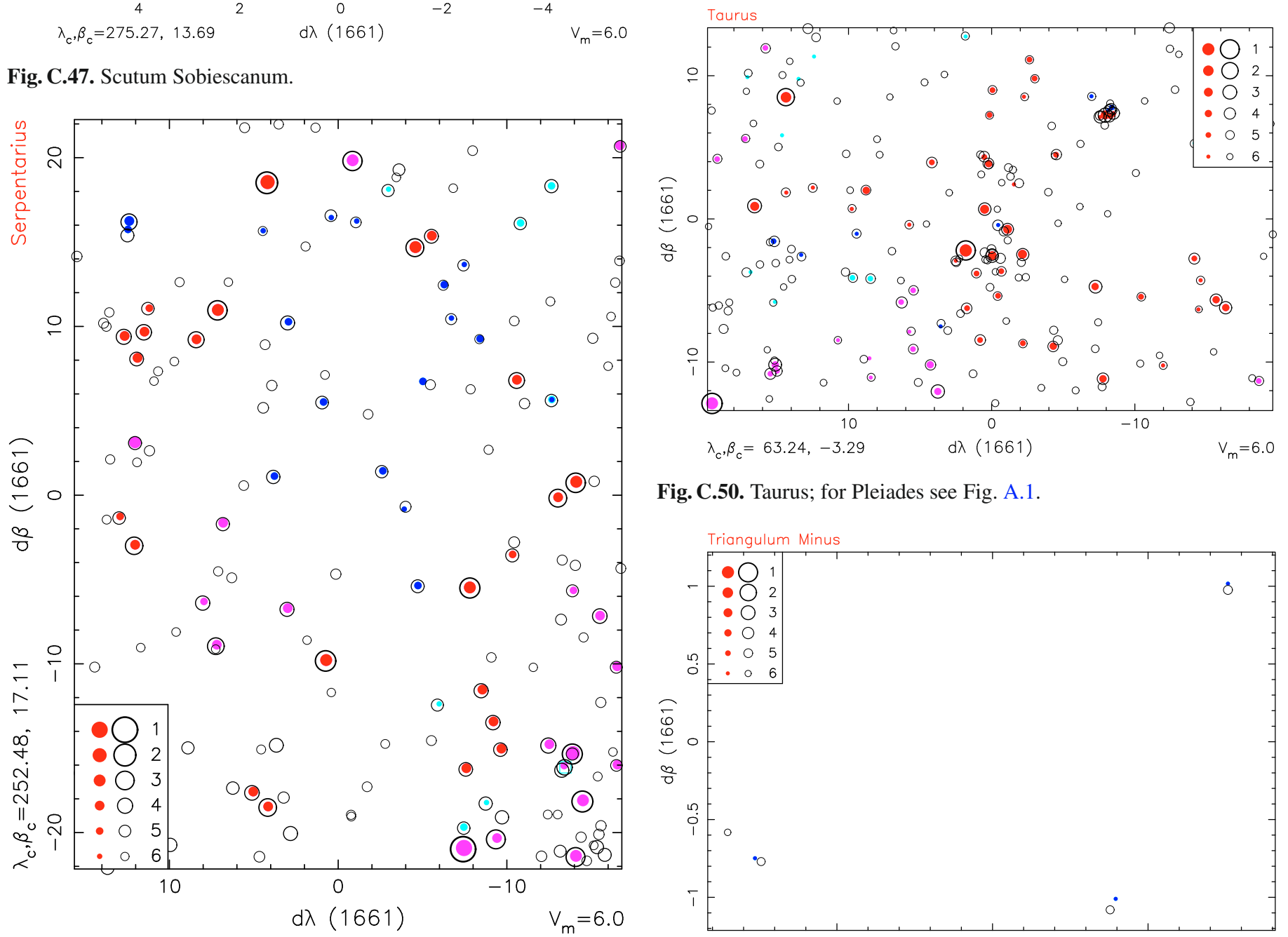

Fig. C.50. Taurus; for Pleiades see Fig. A.1.

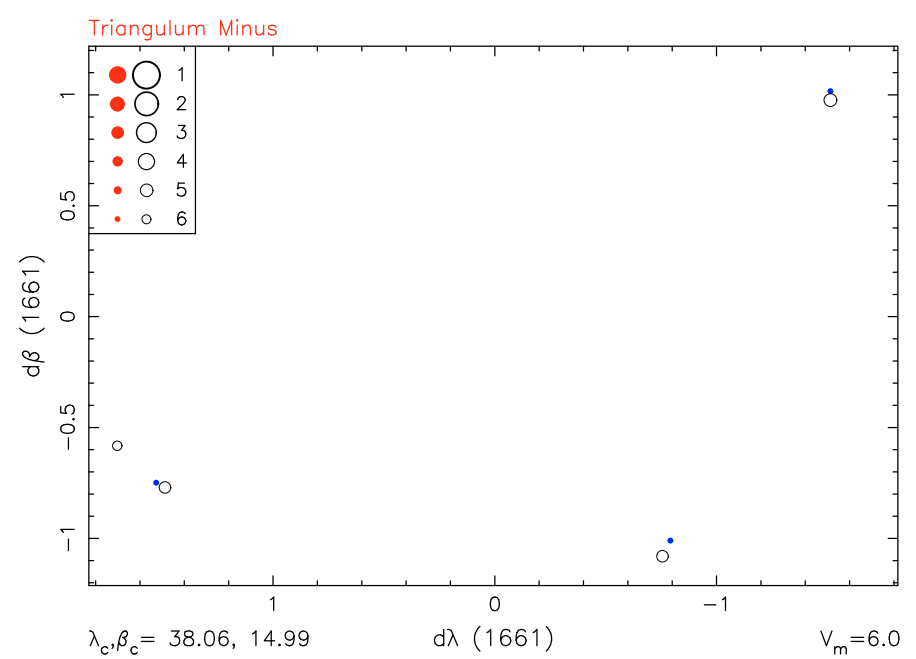

Fig. C.51. Triangulum Minus. 


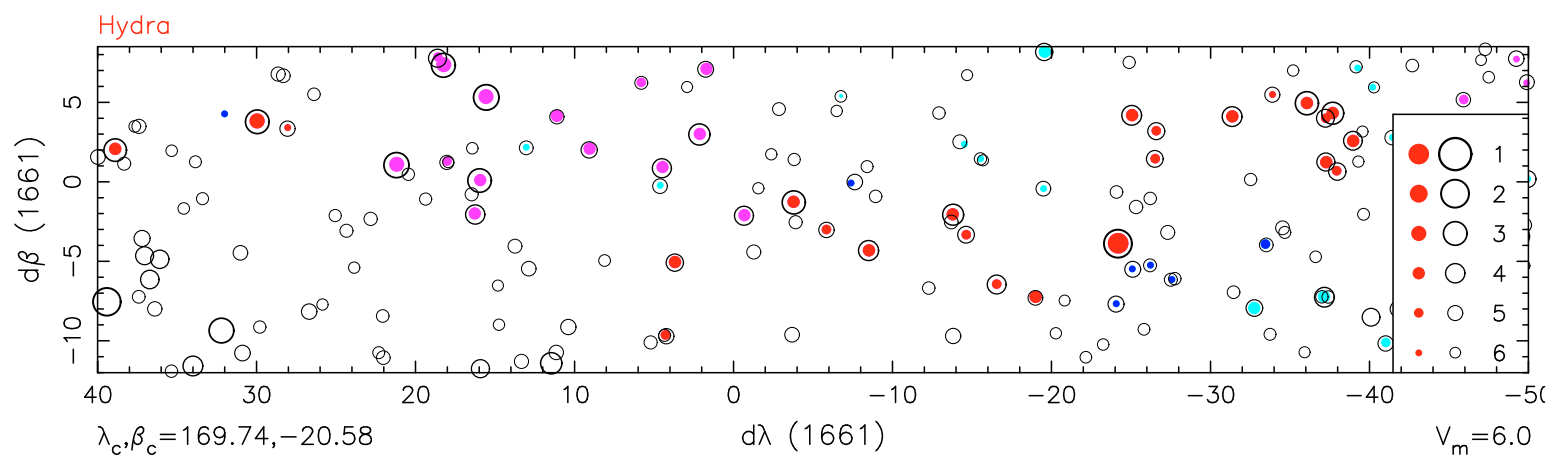

Fig. C.57. Hydra.

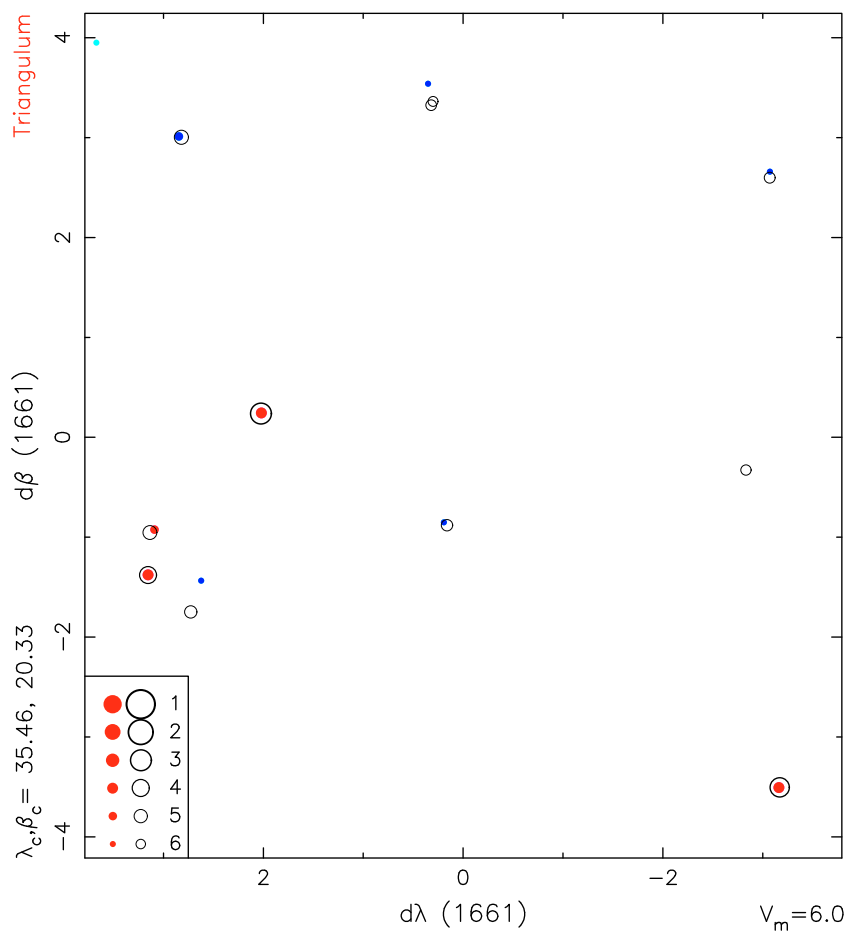

Fig. C.52. Triangulum Maius.

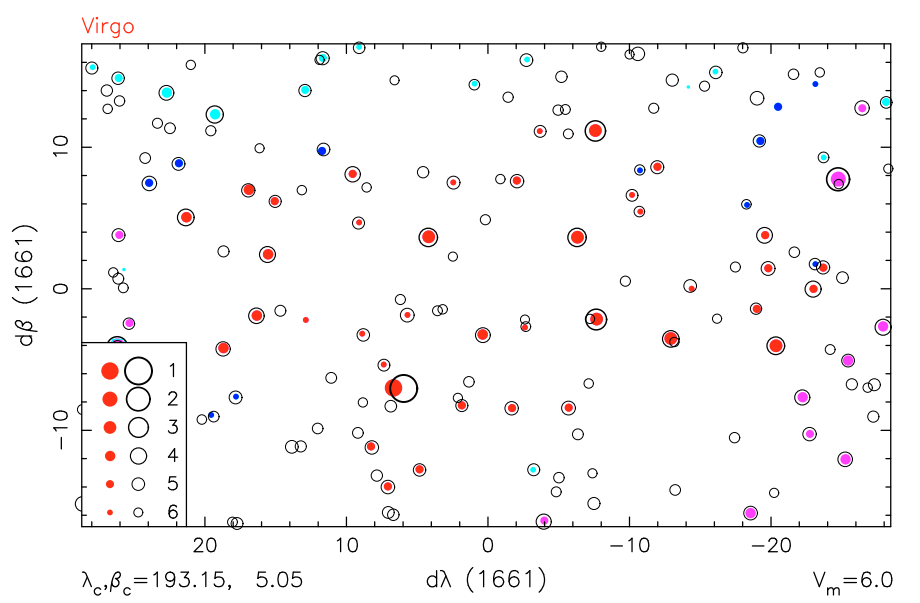

Fig. C.53. Virgo.

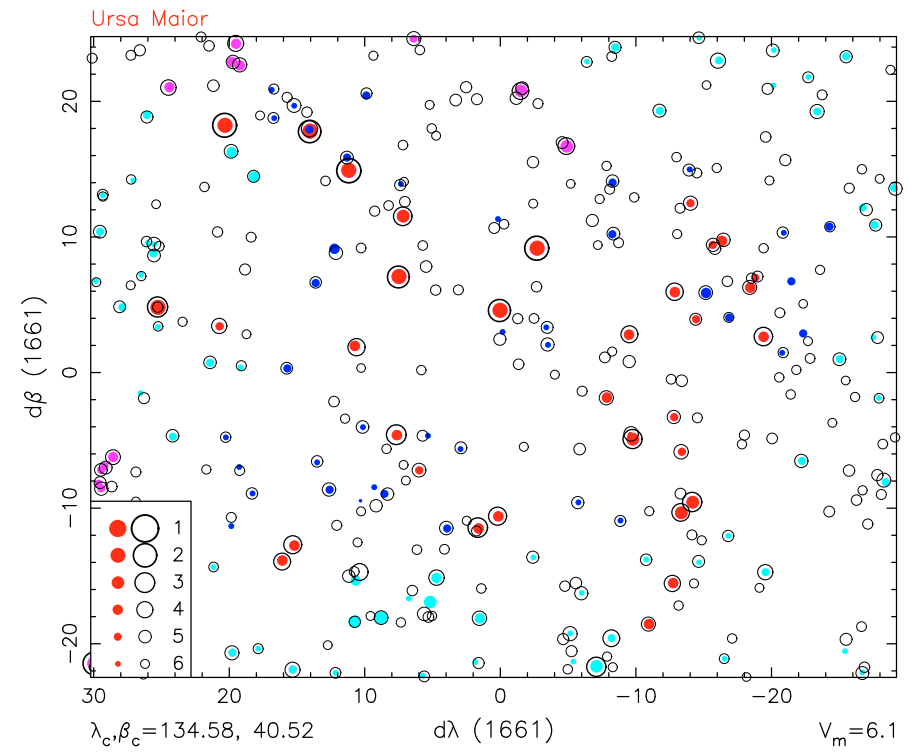

Fig. C.54. Ursa Maior.

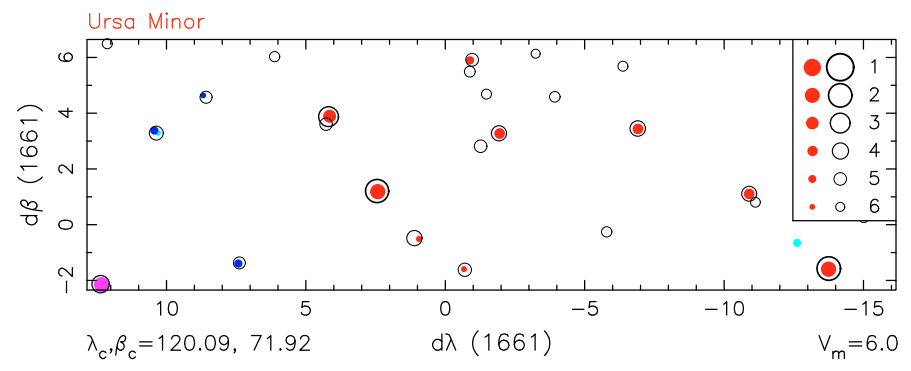

Fig. C.55. Ursa Minor.

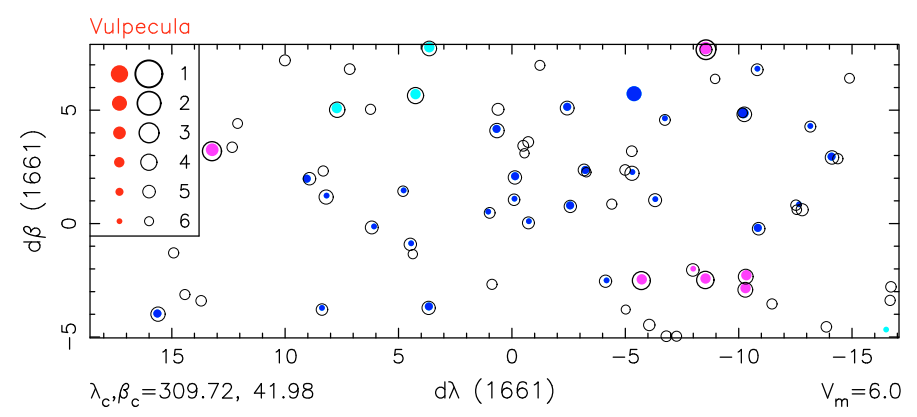

Fig. C.56. Vulpecula. 